University of Nebraska - Lincoln

DigitalCommons@University of Nebraska - Lincoln

Faculty Publications, Department of Physics and Astronomy

Research Papers in Physics and Astronomy

2017

\title{
Superdomain dynamics in ferroelectric-ferroelastic films: Switching, jamming, and relaxation
}

\author{
J. F. Scott \\ University of St. Andrews, jfs4@st-andrews.ac.uk
}

A. Hershkovitz

Technion Israel Institute of technology

Y. Ivry

Technion Israel Institute of technology, ivry@technion.ac.il

H. Lu

University of Nebraska - Lincoln

A. Gruverman

University of Nebraska - Lincoln

See next page for additional authors

Follow this and additional works at: https://digitalcommons.unl.edu/physicsfacpub

Scott, J. F.; Hershkovitz, A.; Ivry, Y.; Lu, H.; Gruverman, A.; and Gregg, J. M., "Superdomain dynamics in ferroelectric-ferroelastic films: Switching, jamming, and relaxation" (2017). Faculty Publications, Department of Physics and Astronomy. 190.

https://digitalcommons.unl.edu/physicsfacpub/190

This Article is brought to you for free and open access by the Research Papers in Physics and Astronomy at DigitalCommons@University of Nebraska - Lincoln. It has been accepted for inclusion in Faculty Publications, Department of Physics and Astronomy by an authorized administrator of DigitalCommons@University of Nebraska - Lincoln. 


\section{Authors}

J. F. Scott, A. Hershkovitz, Y. Ivry, H. Lu, A. Gruverman, and J. M. Gregg

This article is available at DigitalCommons@University of Nebraska - Lincoln: https://digitalcommons.unl.edu/ physicsfacpub/190 


\title{
Superdomain dynamics in ferroelectric-ferroelastic films: Switching, jamming, and relaxation
}

\author{
J. F. Scott, ${ }^{1, a)}$ A. Hershkovitz, ${ }^{2}$ Y. Ivry, ${ }^{2, b)}$ H. Lu, ${ }^{3}$ A. Gruverman, ${ }^{3}$ and J. M. Gregg ${ }^{4}$ \\ ${ }^{1}$ School of Chemistry and School of Physics and Astronomy, University of St. Andrews, St. Andrews KY16 9ST, \\ United Kingdom \\ ${ }^{2}$ Department of Materials Science and Engineering, Technion, Haifa 3200003, Israel \\ ${ }^{3}$ Department of Physics and Astronomy, University of Nebraska, Lincoln, Nebraska 68588, USA \\ ${ }^{4}$ Department of Physics and Mathematics, Queens University, University Rd., Belfast BT7 INN, \\ United Kingdom
}

(Received 21 September 2017; accepted 17 October 2017; published online 1 November 2017)

\begin{abstract}
Recent experimental work shows that ferroelectric switching can occur in large jumps in which ferroelastic superdomains switch together, rather than having the numerous smaller ferroelectric domains switch within them. In this sense, the superdomains play a role analogous to that of Abrikosov vortices in thin superconducting films under the Kosterlitz-Thouless framework, which control the dynamics more than individual Cooper pairs within them do. Here, we examine the dynamics of ferroelastic superdomains in ferroelastic ferroelectrics and their role in switching devices such as memories. Jamming of ferroelectric domains in thin films has revealed an unexpected time dependence of $\mathrm{t}^{-1 / 4}$ at long times (hours), but it is difficult to discriminate between power-law and exponential relaxation. Other aspects of this work, including spatial period doubling of domains, led to a description of ferroelastic domains as nonlinear processes in a viscoelastic medium, which produce folding and metastable kinetically limited states. This $1 / 4$ exponent is a surprising agreement with the well-known value of $1 / 4$ for coarsening dynamics in viscoelastic media. We try to establish a link between these two processes, hitherto considered unrelated, and with superdomains and domain bundles. We note also that high-Tc superconductors share many of the ferroelastic domain properties discussed here and that several new solar cell materials and metalinsulator transition systems are ferroelastic. Published by AIP Publishing.
\end{abstract}

https://doi.org/10.1063/1.5005994

\section{TABLE OF CONTENTS}

I. INTRODUCTION

II. DOMAIN SPATIAL PERIOD DOUBLING

AND TRIPLING.

III. DOMAIN WALL JAMMING RELAXATION: POWER-LAWS OR EXPONENTIAL DECAY?.

IV. SWITCHING OF SUPERDOMAINS ........

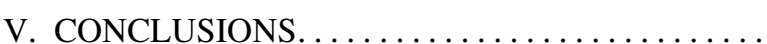

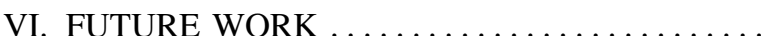

\section{INTRODUCTION}

Domains in ferroelectrics and ferromagnets have been studied for nearly a century now, but several ideas have been emphasized only very recently: These include nonlinear behavior, such as wrinkling, folding, coarsening, and jamming. ${ }^{1}$

\footnotetext{
a)jfs4@st-andrews.ac.uk

b) Present address: Solid State Institute, Technion, Haifa 3200003, Israel.

Electronic mail: ivry@technion.ac.il.
}

In a wide area of physics, ranging from polymer science to geology, the question arises of whether data for a position $\mathrm{x}(\mathrm{t})$, such as a domain wall, can best be described by powerlaw relaxation

$$
\mathrm{x}(\mathrm{t})=\mathrm{A}(\mathrm{t} / \tau)^{-\mathrm{n}}
$$

which is sometimes historically referred to as the Nutting Equation $^{2}$ or to an exponential decay

$$
x(t)=A \exp (-t / \tau)
$$

or a stretched exponential decay

$$
\mathrm{x}(\mathrm{t})=\mathrm{B}[\exp (-\mathrm{t} / \tau)]^{\beta},
$$

which is sometimes referred to as Kohlrausch-WilliamsWatts relaxation, ${ }^{3}$ in which the early work of Kohlrausch was later shown by Williams and Watts to be applicable to polymers.

The conclusion of this review is that these questions are now being asked with regard to relaxation of ferroelectric and ferroelastic domain walls in thin films, and we suggest that these data are yet insufficient to make unambiguous 
discriminations. Moreover, as shown in the references discussed below, especially the work of Kubát and Rigdahl and the work of Weron, for short times, the stretched exponential description may generally prevail, whereas for long times, a simple power-law is asymptotically obtained.

There may be a connection between these relaxation studies in domains and coarsening.

Coarsening is a phenomenon in which grains in ceramics or metals enlarge with time. It is known to exhibit a temporal dependence given typically by $\mathrm{t}^{-1 / 4},-4$ especially at long times $(10-1000 \mathrm{~s}) .{ }^{5,6}$ Nonlinear creep of polarization $\mathrm{P}(\mathrm{t})$ is also found to give an exponent of $1 / 4$ in PZT at low stress, ${ }^{7}$ where the authors refer to this as the Andrade power law. ${ }^{8-10}$ The idea of coarsening has not often previously been extended to wrinkles and domains, rather than grains, but except for very short times the dynamics may be similar in the two cases: ${ }^{11,12}$ Put simply, a different kind of coarsening creates superdomain "bundles" which display both ferroelastic and ferroelectric properties. ${ }^{13}$ In Refs. 11 and 12, Chen and Chen et al. describe the coarsening of wrinkles in viscoelastic layers and the jamming in magnetic domains; and in Refs. 14-17, Salje et al. and Zvelindovsky et al. describe the coarsening in multidomain systems. They also show that shearing slows down coarsening of structures at later stages of phase separation, trapping the system in kinetically driven non-equilibrium metastable states. We note that Salje uses the term "coarsening" to refer to the broadening of crossed ferroelastic domains in a fine "tweed" structure to a coarser "tartan" structure. ${ }^{15}$

Salje has emphasized that these tweed structures can form a domain glass with a nonergodic response to external forcing. Such domain glasses can contain polar nanoregions, which are better known to exist in relaxor materials. He points out that complex domain structures, including tweed, may be stabilized by defects and that tweed structures are polar, either via the flexoelectric effect or via bilinear coupling between the strain and local dipole moments. Recently, Salje et al. ${ }^{16}$ reported the first experimental evidence for piezoelectricity of a tweed structure in $\mathrm{LaAlO}_{3}$, where the uniform parent structure is centrosymmetric and shows no bulk polarity.

Both Chen and Zvelindovsky et al. found ${ }^{12-18}$ powerlaw dependences with exponents very near $1 / 4$, as does Sinha. ${ }^{14}$ There is a good earlier study of this problem by Kohn and Otto ${ }^{19}$ based upon surface diffusion. A slightly different temporal exponent of $-1 / 3$ is obtained in the earlier model(s) of Wagner ${ }^{20}$ and of Lifshitz and Slyozov. ${ }^{21}$ To put things into recent perspective, however, as recently as 2011, it has been determined ${ }^{22}$ that "there is yet no physical interpretation for the loss mechanism" describing relaxation of 90-degree walls in the most popular ferroelectric, lead zirconate-titanate (PZT). This agrees with the earlier opinion on exponential versus power-law relaxation ${ }^{23}$ that "Current theories of solid state flow cannot provide an explanation of the $v \sigma$-behavior observed." Here, $v$ is the activation volume and $\sigma$ is the stress. These authors suggest an empirical relationship, such that power-law relaxation occurs in both metals and polymers only when $v \sigma>10 \mathrm{kT}(250 \mathrm{meV}$ at ambient T) and exponential decay for smaller stresses. This behavior may be compared with the limit in magnetic domain relaxation mentioned below that depends upon the ratio of dipolar to exchange energies.

Jamming and coarsening of domains are not equivalent phenomena, since in jamming the average size of the domains need not change; that is, very small domains are not necessarily annihilated to be incorporated into larger domains, although average domain areas may increase with time to minimize perimeters. Conversely, coarsening can occur for systems such as solid grains in a liquid or glassy matrix in which the grain boundaries rarely touch each other (Ostwald ripening ${ }^{24}$ ). Therefore, it is useful to examine carefully over what range of time, temperature, stress, electric field, or local electro-chemical ionic distribution the dynamics of domain coarsening and jamming are similar. Very recently, the relationship between coarsening and jamming has been examined in the special case of foams. ${ }^{25}$ Both coarsening and jamming arise to minimize the surface energy at the domain walls. In the case of coarsening, the domain wall area $(A)$ increases, while specific surface energy $(\gamma)$ does not change

$$
\Delta G=\gamma \cdot \Delta A
$$

That is, coarsening takes place only when there is no change in the domain wall energy during the domain wall motion. In the more general case, the domain wall energy itself can also vary

$$
\Delta G=\Delta(\gamma \cdot A) .
$$

For example, due to the change in defects or pinning sites that arise due to ferroelastic domain wall reorganization. ${ }^{26}$

A second question to consider is whether coarsening in multiferoics leads to superdomains or "bundles." Figure 1 (left) shows a typical ferroelectric with 180-degree in-plane domains in which polarization $\langle\mathrm{P}\rangle$ averages to zero, but strain $\langle\mathrm{S}\rangle$ is non-zero (differing significantly along or perpendicular to the polarization in each layer). Figure 1 (right) shows the converse effect involving in-plane 90-degree domains, in which $\langle\mathrm{S}\rangle$ averages to zero, but $\langle\mathrm{P}\rangle$ is nonzero. In very early years (1955), it was known that some sort of superdomain structures was formed in barium titanate; Fig. 2 illustrates ${ }^{27}$ such a case. In many ferroelectric-ferroelastics, superdomains will form in which $\langle\mathrm{P}\rangle$ and $\langle\mathrm{S}\rangle$ both average to zero over mesoscopic distances, to minimize depolarization and strain energies (Fig. 3). ${ }^{28-30}$ In other systems, especially smectics, it has been shown that nano-ferroelectric domains constrained inside such ferroelastic domains can be normal to the ferroelastic walls or tilted (as in Ref. 28), with a possibility of a phase change from one to the other in between the ferroelastic walls. ${ }^{29,30}$

We shall term these arrays "superdomains," although some authors term them "bundles." "31 The simplest of these structures are closure domains in which typically four structures of the type illustrated in Fig. 1 are arranged clockwise or counterclockwise in a closed group. These have been studied in some detail previously. ${ }^{13,32-34}$ In particular, we know that these arrays can be moved via an electron beam in an electron microscope ${ }^{35}$ and that they exhibit creep. ${ }^{36,37}$ 


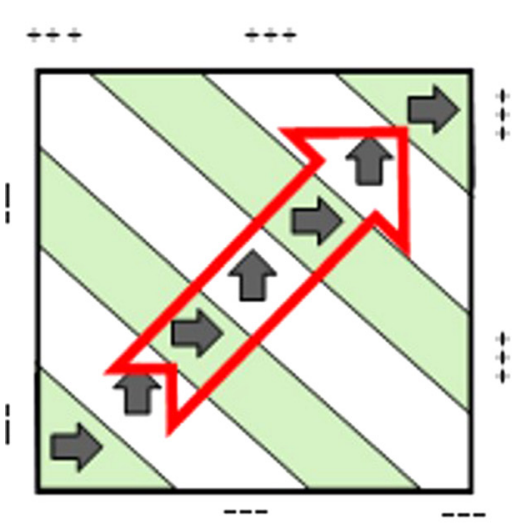

No macroscopic shape change BUT resultant macroscopic polarisation

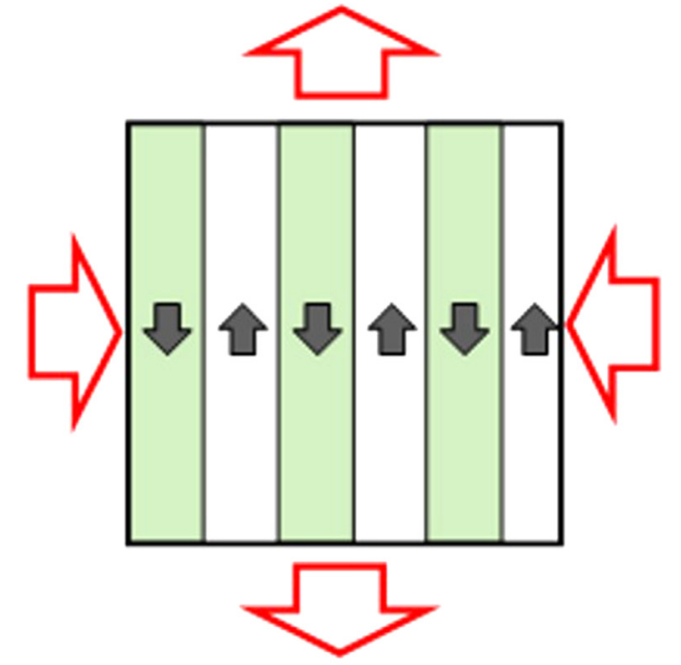

No macroscopic polarisation BUT resultant shape change
FIG. 1. (a) Right-hand-side: 180degree in-plane domains with average polarization $\langle\mathrm{P}\rangle=0$ but nonzero average strain $\langle\mathrm{S}\rangle$ (indicated by large arrows); (b) Left-hand-side: 90-degree in-plane domains with average strain $\langle\mathrm{S}\rangle=0$ but nonzero average polarization $\langle\mathrm{P}\rangle$, indicated by large arrow. This stress will produce domain wall motion. Both herringbone and closure domain structures produce $\langle\mathrm{S}\rangle=\langle\mathrm{P}\rangle$ $=0$.
It is often found that ferroelectric nano-domains are nested inside larger ferroelastic domains such that the polarization (and hence depolarization energy) averages mesoscopically to zero; but the opposite case, of ferroelastic domains nested inside larger ferroelectric domains seems rare. (A good exception, however, is ${ }^{38,39} \mathrm{Sr}_{\mathrm{x}} \mathrm{Ba}_{1-\mathrm{x}} \mathrm{Nb}_{2} \mathrm{O}_{6}$ with $0.60<\mathrm{x}<0.75$ with nano-ferroelastic domains clustered around the fourfold channels in this tungsten bronze, inside 300-nm-diameter ferroelectric domains; and a more recent exception is the spinel $\mathrm{GaV}_{4} \mathrm{~S}_{8}$, where skyrmion-like ferroelastic domains of diameter $20 \mathrm{~nm}$ are found inside ferroelectric domains an order of magnitude larger. ${ }^{40}$ ) One might ask why, that is, why does the strain energy not often also average to zero in the same way? We believe that the answer is that strain is never screened, whereas charge and polarization are for the relevant length scale. In this respect, it is probably important that $\mathrm{GaV}_{4} \mathrm{~S}_{8}$ is a semiconductor, or more precisely a Mott insulator, ${ }^{41}$ so that screening differs in it compared with a typical more insulating oxide ferroelectric and that the ferroelectric transition is order-disorder and not displacive.

In addition to systems in which ferroelastic domains are all larger than the ferroelectric domains or are all smaller, there are some materials such as $\mathrm{WO}_{3}$ that exhibit a whole hierarchy of ferroelastic length scales. ${ }^{42}$ In that crystal, there are two different coexisting domain widths, thin around pseudocubic $\langle 100\rangle$ axes and thick around $\langle 110\rangle$, neither of which satisfies the Landau-Lifshitz-Kittel Law for thickness

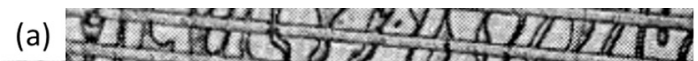

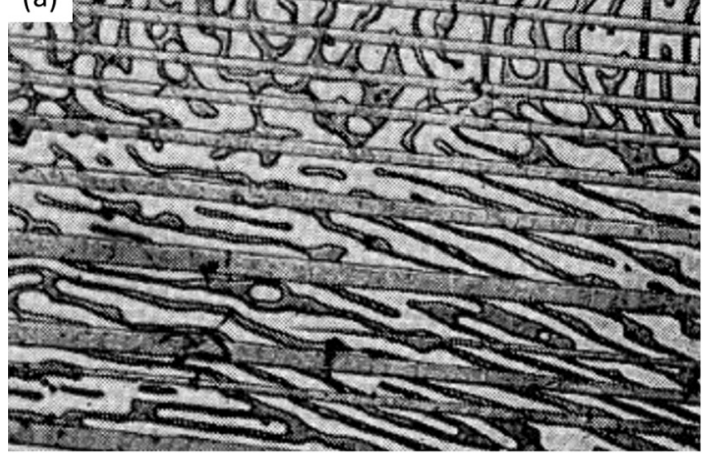

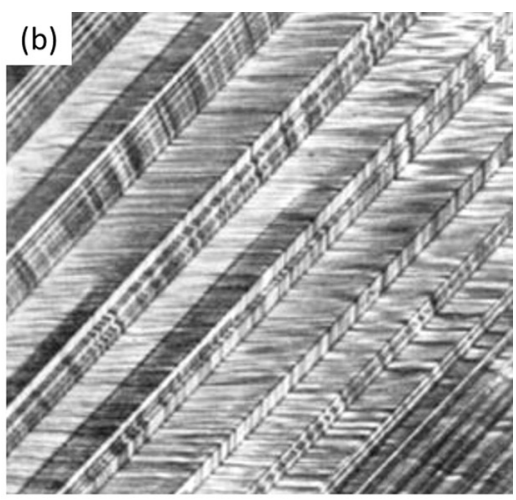

(c)

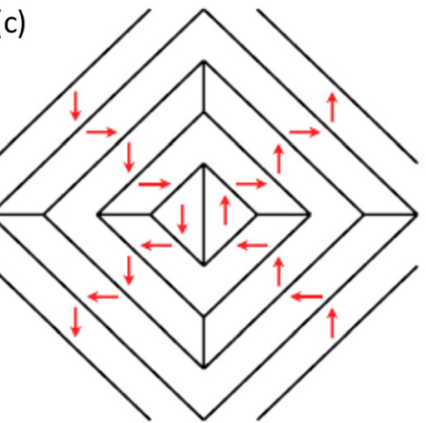

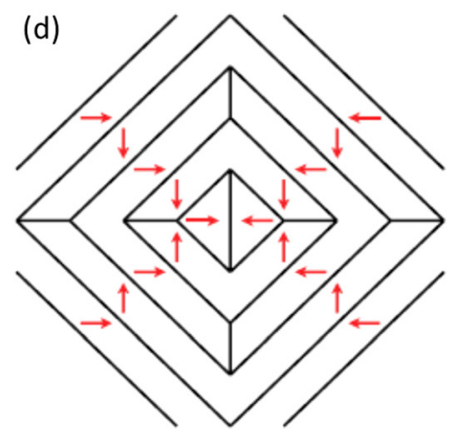

FIG. 2. (a) A real superdomain from early work on $\mathrm{BaTiO}_{3}$ : Stacks of flat ferroelastic 90-degree domains order with wavy 180-degree ferroelectric domains inside them. Note that some of the wavy domains are constrained by flat domain walls and some interpenetrate those walls. The 180-degree domain walls are very straight normal to the plane of the figure but hang in folds like drapes within the plan view. (b) Large ferroelastic domains each containing small ferroelectric nanodomains. ${ }^{27}$ Reprinted with permission from J. A. Hooton et al., Phys. Rev. 98, 409-413 (1955). Copyright 1955 American Physical Society. (c) Quadrupolar and (d) radial patterns of two possible arrangements of domains. 

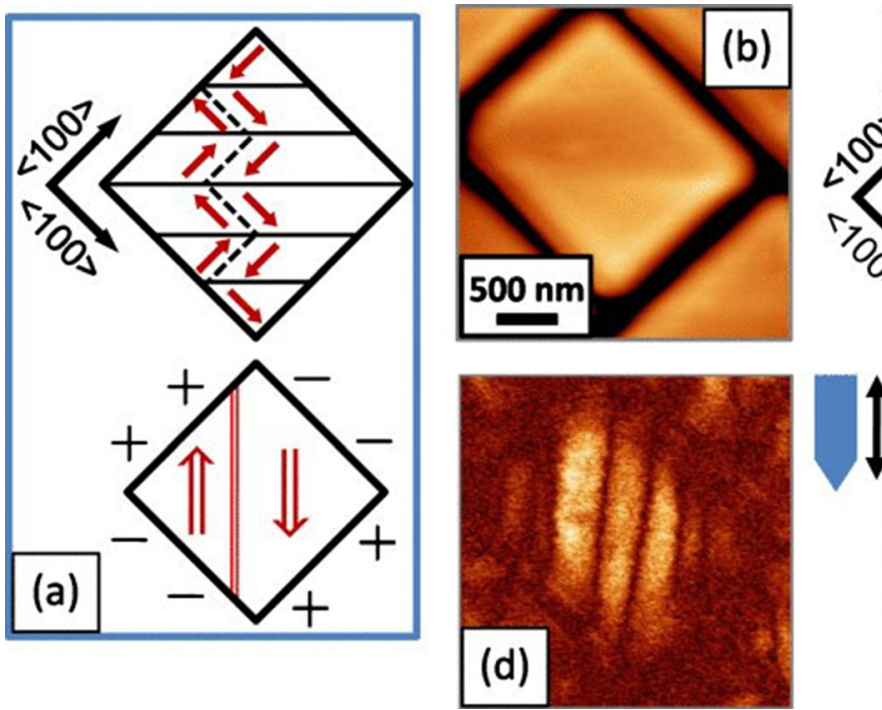

dependence on the film thickness to the power of 0.5: The thin domains vary to the power of 0.6 , and the thick ones to the power of 0.4 (so that at asymptotically thick values, they become equal!).

Parenthetically, we note that these domain walls can be either charged or neutral. Janovec showed 40 years ago ${ }^{43}$ that at the ferroelectric-paraelectric phase transitions, charged walls usually arise where there had been mirror planes and uncharged walls, where there had been twofold axes. In addition to these two kinds of ferroelastic walls (mirror plane and twofold axis), more recently, it has been shown that ferroelastic fourfold axes also exist. ${ }^{44}$

These ferroelastic bundles switch as a block in some ferroelectrics, ${ }^{45,46}$ resulting in hysteresis loops with very large steps. We emphasize this aspect of superdomains, because it will control ferroelectric switching in many thin-film devices, because most ferroelectric memory materials are also ferroelastic. In these superdomains, the interactions among the ferroelastic domains and domain walls control the switching dynamics, not the behavior of smaller ferroelectric domains inside each large ferroelastic cluster or bundle. This behavior is similar to the dominancy of vortex-antivortex interactions over the Cooper-pair interactions in Type II superconducting confined geometries as described by the Kosterlitz-Thouless model. ${ }^{47}$ Very recently, it has been pointed out that surface creases in elastic films are analogous to Kosterlitz-Thouless instabilities. ${ }^{48}$ Other authors have shown ${ }^{49,50}$ that Kosterlitz-Thouless melting within n-vertex Potts model descriptions requires vertices with $n>4$ and does not arise from the threefold and fourfold vertices considered by Srolovitz and $\mathrm{Scott}^{51}$ and characteristic of $\mathrm{BaTiO}_{3}, \mathrm{PbTiO}_{3}$, and PZT films considered here. We should note that our analysis is supported by a recent work by Sigov who suggested that defects in ferroelectrics can be correlated and comply with the Kosterlitz-Thouless model. ${ }^{52}$ In the Kosterlitz-Thouless framework, the energy of system is determined by the vortex-antivortex energy binding, $\rho$. This energy represents also the superfluidic stiffness, i.e., the viscosity or vorticity of the system. In analogy, the ferroelasticferroelastic interactions in bundle domains also represent the

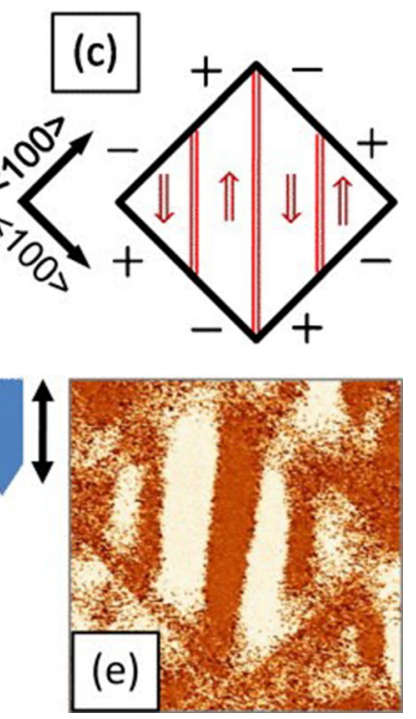

FIG. 3. A bundle or superdomain ${ }^{84}$ that minimizes both strain energy and depolarization energy by forming a mesoscopic array with both $\langle\mathrm{P}\rangle=\langle\mathrm{S}\rangle=0$ : (a) Top: $\langle\mathrm{S}\rangle=0$ state in the core; bottom: $\langle\mathrm{P}\rangle=0$ state in the core. The top and bottom configurations do not have the same total energy. The bottom seems preferred in our materials, probably because charge is screened but strain is unscreened. (b) Experiment. (c) $\langle\mathrm{P}\rangle$ $=0$ state at the core with spatial period halved in comparison with (a). (d) and (e) Experimental data. Reprinted with permission from Appl. Phys. Lett. 98, 132902 (2011). Copyright 2011 AIP Publishing LLC. viscosity of the system. We suggest that it is this rheological characteristic of the ferroelastic domain network in superdomains that gives rise to doubling, tripling, and wrinkling.

A simple way to pose this question is to consider the time evolution of such bundles as a ferroic film is cooled below its Curie temperature, $\mathrm{T}_{\mathrm{c}}$. Do these extended arrays form to minimize strain and depolarization energy in thermal equilibrium? Are they non-equilibrium structures that are kinetically limited?

A series of studies of ferroelastic domain walls has been published by Salje, of which the recent examples are given in Refs. 53-55. These generally describe the kinetics via stretched exponentials rather than power laws. They do, however, introduce nonlinearities in the context of avalanches. Moreover, they show ${ }^{56}$ that the jamming relaxation follows a power law at low temperatures but an exponential at higher temperatures. A similar conclusion was reached by Metzler and Klafter, who obtain stretched exponentials and power laws as the two asymptotical limits of a MittagLeffler equation. ${ }^{57,58}$ A good study of the interaction of ferromagnetic, ferroelectric, and ferroelastic domains has been given recently by Van de Wiele et al. ${ }^{59}$

It is more common to treat relaxation data for dielectrics to a stretched exponential, rather than a power law. However, Weron shows that the stretched exponential is merely a special limiting approximation to a more general power law. ${ }^{60-62}$ She points out that the exponential arises when one neglects the distribution of waiting times for the relaxing dipoles and when one treats each relaxing dipole as independent, as contrasted with cluster behavior. The problem with many switching models (e.g., Avrami and nucleation-frustrated) is that they assume all relaxation starts at the same time $\mathrm{t}=0$; that is, there is no "waiting time." In reality, several different mechanisms can contribute to such waiting time, including back-switching processes; the net result is that most of the relaxation process begins not at $\mathrm{t}=0$ but at a finite delay time $\tau_{0}$. At best, this introduces another fitting parameter to the data, and unfortunately this parameter $\tau_{0}$ will be highly correlated with the exponent used in the least squares fit. 

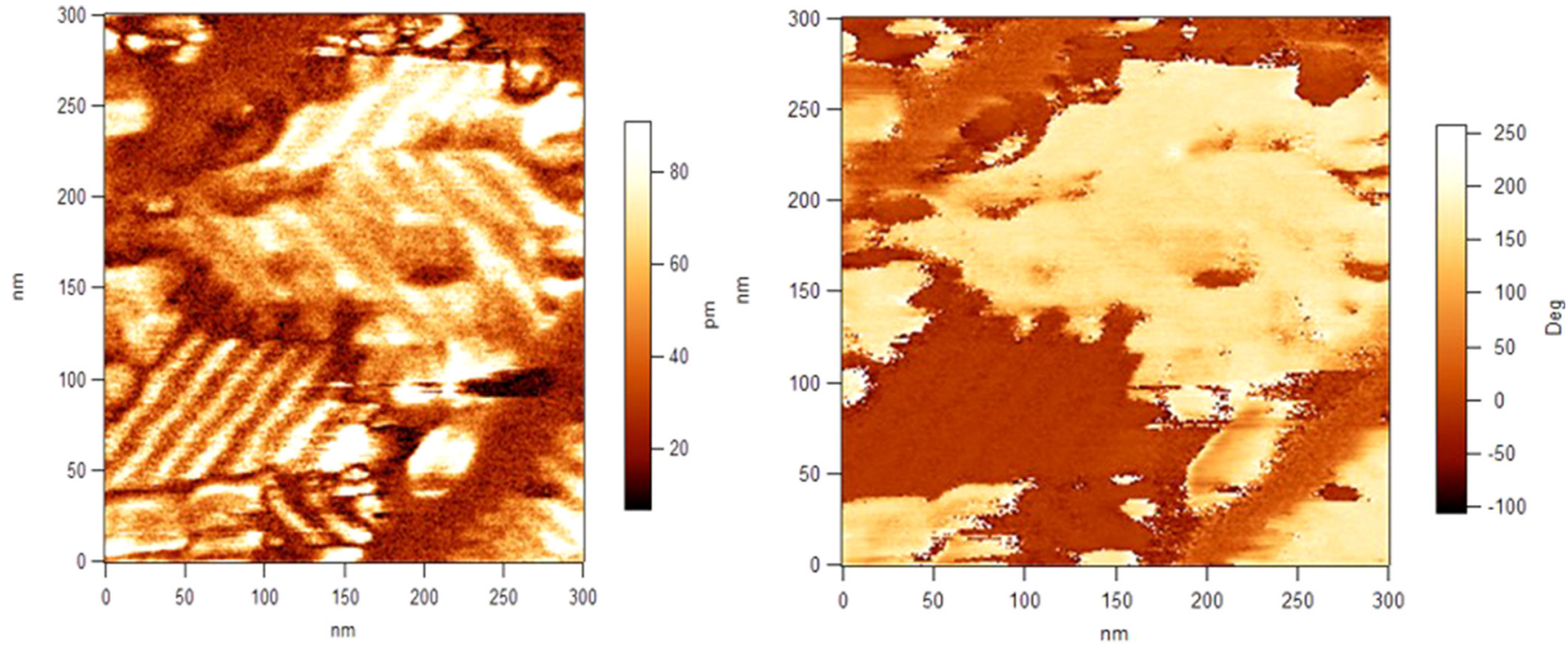

FIG. 4. Lateral PFM amplitude (left) and phase (right) of the 9-nm-thick $\mathrm{PbTiO}_{3}$ film. Note the spatial period doubling near the top, at the chevron-like interface, and in the middle left side. The larger domain stripe widths do not satisfy the Landau-Lifshitz-Kittel Law. ${ }^{88-90}$

Many researchers have shown that these powers are fractional; and it is interesting to note that power-law relaxations in solids date back to the early 18 th century. ${ }^{63}$ For magnetic domains, the relaxation can follow either an exponential decay or a power-law decay, depending upon the ratio of dipolar to exchange interactions. ${ }^{64}$

More recently, the non-Ising qualities of ferroelectric domain walls have been analyzed, and it would appear that some of the mysterious losses and relaxation may involve flexoelectricity, which is always present, independent of crystal symmetry, and estimated in $\mathrm{PZT}$ or $\mathrm{BaTiO}_{3}$ as about $4 \%$ of the total polarization of the domain walls. ${ }^{65,66}$

Finally, readers are referred to an excellent treatise on ferroic domains by Jill Guyonnet for a discussion of these problems. ${ }^{67}$

\section{DOMAIN SPATIAL PERIOD DOUBLING AND TRIPLING}

Reference 1 illustrates period doubling in ferroelastic domains in samples of lead zirconate-titanate iron-tantalate that have been subject to focused-ion beam cutting. There are two questions that might be asked regarding those data: First, does it occur in other ferroelectric/ferroelastics? And second, does it require FIB processing to provide sufficiently nonlinear stresses? Figure 4 answers these questions by showing domain period-doubling (top of figure) in $\mathrm{PbTiO}_{3}$ films (on $\mathrm{KTaO}_{3}$ substrates) that have not been subject to FIB. These are $22 \mathrm{~nm}$ thick. The thinner domains are about $12 \mathrm{~nm}$ wide, and the thicker, about $25 \mathrm{~nm}$. These widths are not in accord with the Landau-Lifshitz-Kittel formula for a $22 \mathrm{~nm}$ thickness, given numerically by Refs. 68 and 69, which further supports their nonlinear and/or non-equilibrium origin.

Figure 5 illustrates a similar period doubling in nonepitaxial, thicker (60-nm) ceramic PZT films. The microscopic, atomistic dynamics are revealed in the dislocation line creation and annihilation shown in Fig. 6. And in Fig. 7, we see a spatial tripling of domain width. Such a tripling was initially predicted in the nonlinear domain wall creep model of Metaxas et al.; ${ }^{70}$ and Wang and Zhao showed ${ }^{71}$ that it requires a higher strain rate than does doubling. The detailed model of Wang and Zhao merits a fuller discussion. Professor Xuanhe Zhao and his postdoc Qiming Wang describe these regimes of wrinkling, folding, and period-doubling as separate thermodynamic phases, with true phase boundaries between them. These authors show how wrinkling in domains evolves into folding and subsequently to period doubling as a function of both stress and the ratio of film/substrate thickness, in a three-dimensional phase diagram. In general, it is possible to go to frequency doubling without folding first (via increased strain) or to go from folding to period doubling directly via decreased film thickness. A qualitative feature of their model

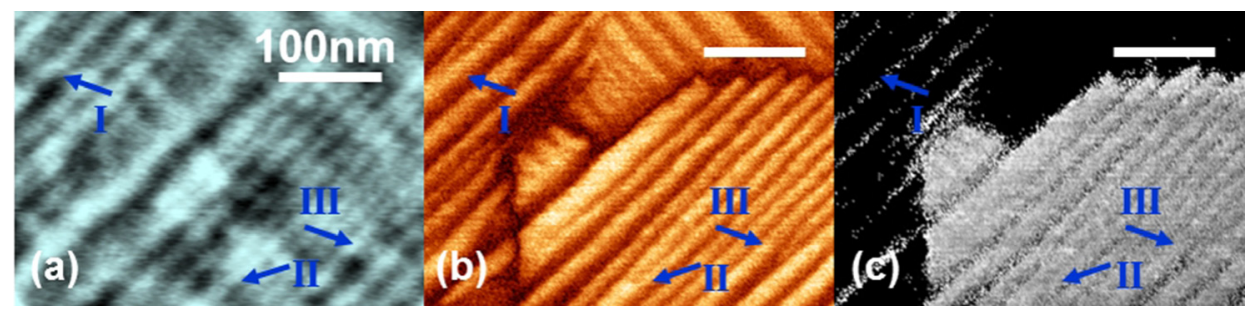

FIG. 5. Atomic force micrographs of spatial period doubling in PZT films [clearest at interface in upper right portion of (b) above; note also the bifurcations marked as II and III): (a) topography; (b) amplitude; (c) phase. Reproduced with permission from Y. Ivry et al., Adv. Funct. Mater. 24, 5567-5574 (2014). Copyright 2014 John Wiley and Sons. 


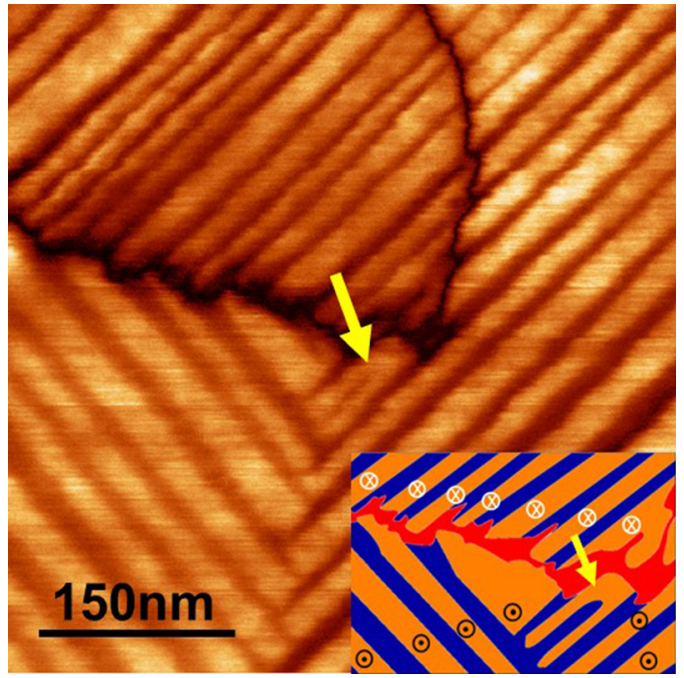

FIG. 6. Details of period doubling within a PZT single grain, showing that the dynamics involve creation or unwinding of a line of dislocations. Larger stripe width is $25 \mathrm{~nm}$. This kind of splitting is common in liquid crystal domains and is referred to as a Bobylev-Pikin flexoelectric instability. ${ }^{91}$

is that the thin film must have a larger modulus than the substrate; it forms a crust which must fold or wrinkle under compression to avoid delamination. Note that it has neither been known, in general, how thick ferroelectric domains are (are they near-surface layers?) with a few exceptions ${ }^{72}$ nor what their shear moduli are.

Not all models or systems exhibit spatial tripling: Brau et $a$. $^{73}$ show that a sequence of doubling, quadrupling, etc., can occur without tripling; such a sequence eventually leads to chaos. The same group ${ }^{74}$ has shown that the period doubling is a continuous second-order phase transition and that it requires an up-down asymmetry; hence it is not present for their model in sandwich-structure thin films in which the lower substrate and top capping layer are identical.

\section{DOMAIN WALL JAMMING RELAXATION: POWER- LAWS OR EXPONENTIAL DECAY?}

Some sparse data on relaxation of domains in other ferroelastics exist (Fig. 8). Figure 8 illustrates data on the decay of surface potential around 90-degree walls in single-crystal
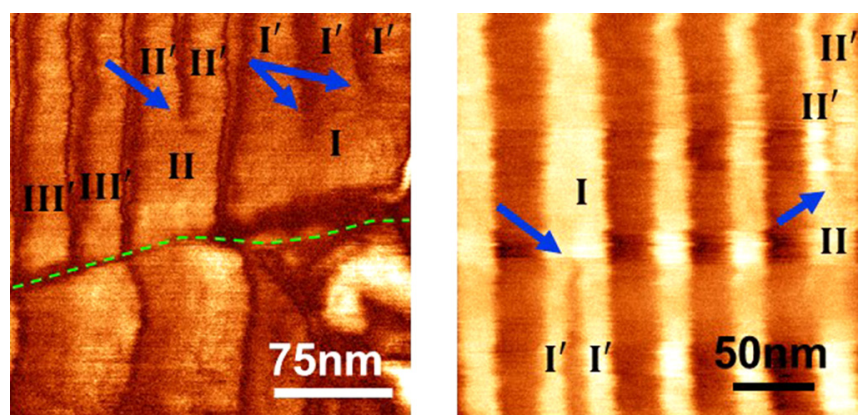

FIG. 7. (Left) Spatial tripling in PZT films (left edge of micrograph), predicted by Metaxas et al. ${ }^{49}$ and Wang and Zhao. ${ }^{50}$ Tripling is also illustrated in the PFM micrograph on the right hand side (upper right corner). ${ }^{75}$ Reproduced with permission from Y. Ivry et al., Adv. Funct. Mater. 21, 1746 (2011). Copyright 2011 John Wiley and Sons. ${ }^{123}$ (Right) spatial doubling, marked in blue arrows, in two (I-II) close by elastic domains.
$\mathrm{BaTiO}_{3} \cdot{ }^{75-77}$ The fits to exponential decay with $\tau=30$ s and with a power law are both shown, illustrating the difficulty in unambiguous discrimination for typical published data. Note that for power laws with exponent $\mathrm{n} \ll 1$, any data asymptotically approach a logarithmic (exponential) dependence.

These show that the relaxation time for 90-degree walls is ca. $100 \times$ faster than for 180 -degree walls. ${ }^{70}$ For 90 -degree walls in $\mathrm{PbZr}_{0.3} \mathrm{Ti}_{0.7} \mathrm{O}_{3}$, the relaxation time is typically $780 \mathrm{~s}$, whereas for 180 -degree walls, it is ca. $10^{5} \mathrm{~s}^{78}$ This compares with a jamming relaxation time of ca. $5000-7000 \mathrm{~s}$ in $\mathrm{BaTiO}_{3},{ }^{32,33}$ suggesting that the jamming time (almost the same as 180 -degree reversals) is not limited primarily by 90 degree wall relaxation. The diameter of the typical bundle or superdomain studied in Ref. 75 was $80 \mathrm{~nm}$. This gives a ferroelastic area that typically contains ca. 100 ferroelectric nano-domains (see Fig. 9, bottom diagram). The fact that the ferroelectric domains can be reoriented within a larger ferroelastic superdomain by the application of electric field has been demonstrated by electron beam irradiation ${ }^{34,35}$ with faceting and de-faceting observed.

There are three fundamentally different non-equilibrium patterns for domains: The one in the upper left panel of Fig. 4 is usually termed a dislocation pattern; and the one in the lower left panel of Fig. 9 is called a spiral pattern. These have quite different dynamical origins, discussed in theoretical detail by Hohenberg and Krekhov, ${ }^{79}$ who show that the dislocation pattern arises from their "Type I.s stationaryperiodic instability," whereas the spiral pattern arises from their "Type III.o oscillatory-uniform instability;" they also show a third "target pattern" shaped like a bulls-eye, which was reported in ferroelectrics by Gruverman et al. and by Dawber et al. ${ }^{80,81}$ and called a "vortex pattern" or "perimeter effect" as shown in Fig. 9(c). In addition, they show a "domain boundary pattern" that can include "herringbone" configurations. They comment that in this categorization the dislocation patterns have a mechanism similar to that in Rayleigh-Benard convection instabilities or Taylor-Couette instabilities with rotating inner cylinders, whereas the spiral pattern is related to the Belousov-Zhabotinsky reaction instability.

One should point out that the number of sides of the domains in jammed ferroelastic-ferroelectrics are not random but are influenced by the strong preference for vertices to be threefold in the materials considered. ${ }^{51}$ This severely constrains the available geometries. Hence, although jamming is sometimes described as chaotic, it is not geometrically random.

Finally, we note that the reverse of Ostwald ripening and jamming has been studied in magnets when the applied field is turned off: There is a decrease in size of domains and an increase in the number of domain walls as magnetization flops from out-of-plane to in-plane. ${ }^{82}$

\section{SWITCHING OF SUPERDOMAINS}

Figure 10 shows the TEM photos of a ferroelastic superdomain switching large amounts of charge. ${ }^{45,83}$ This shows that ferroelastic superdomains move as large coherent blocks 
(a)

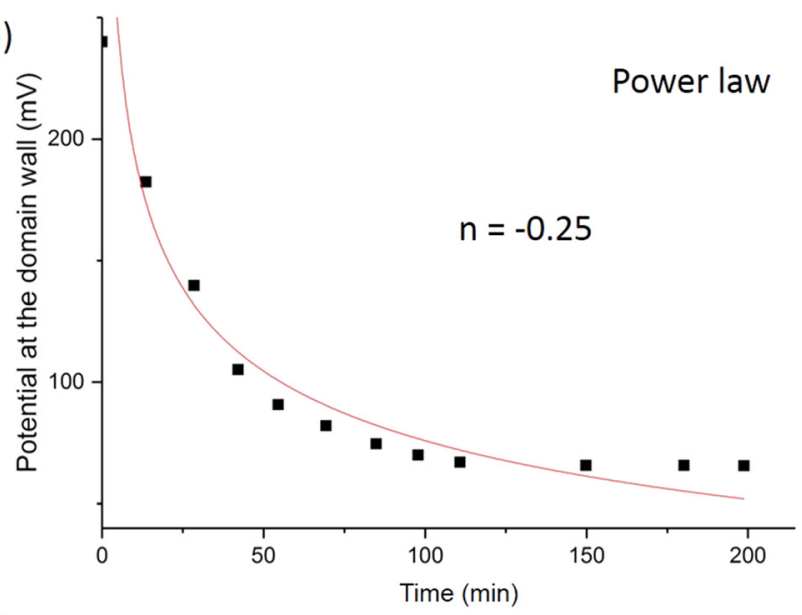

(c)

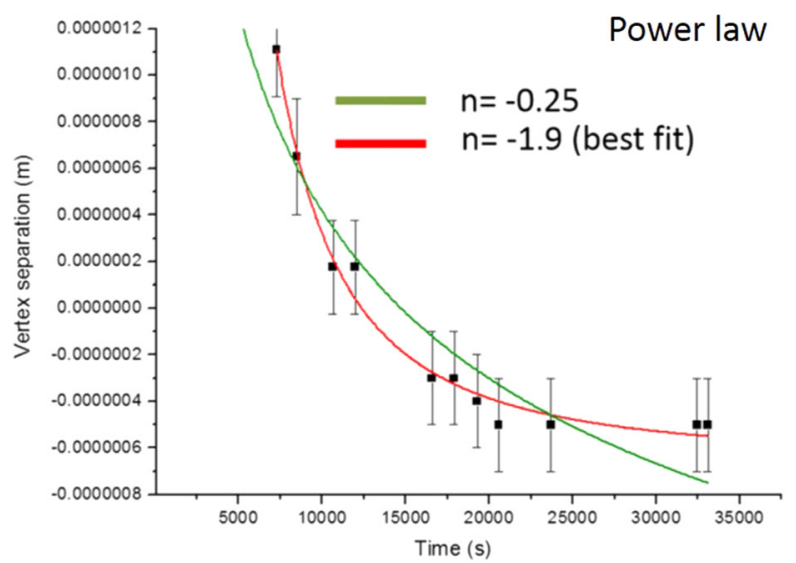

(e)

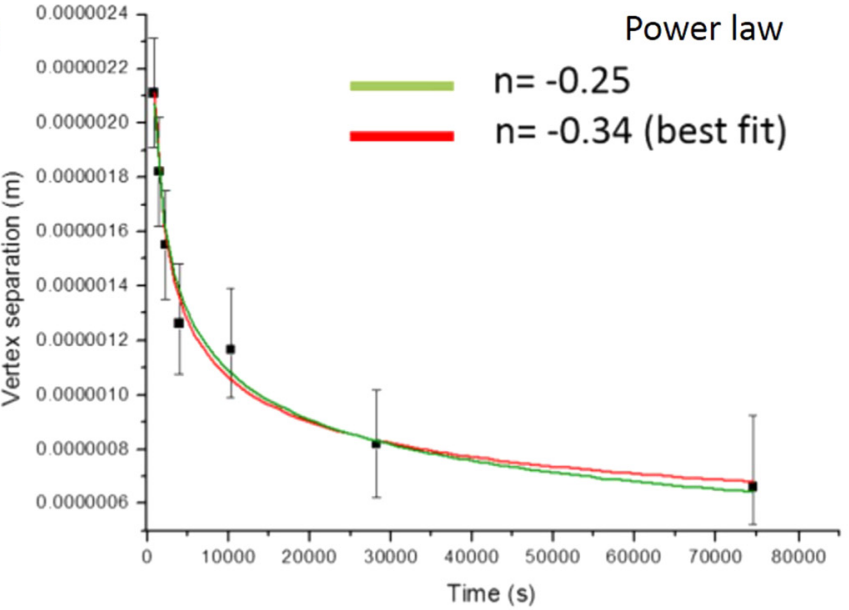

(b)

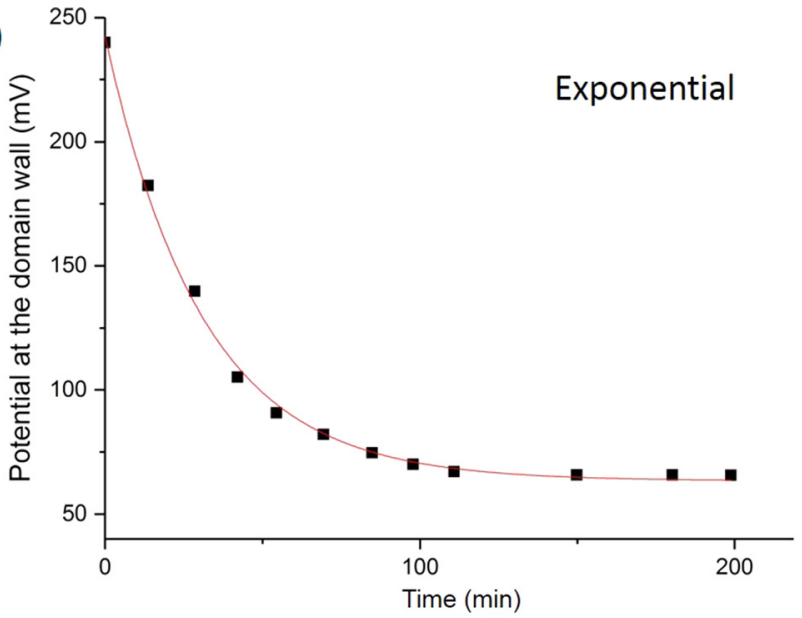

(d)

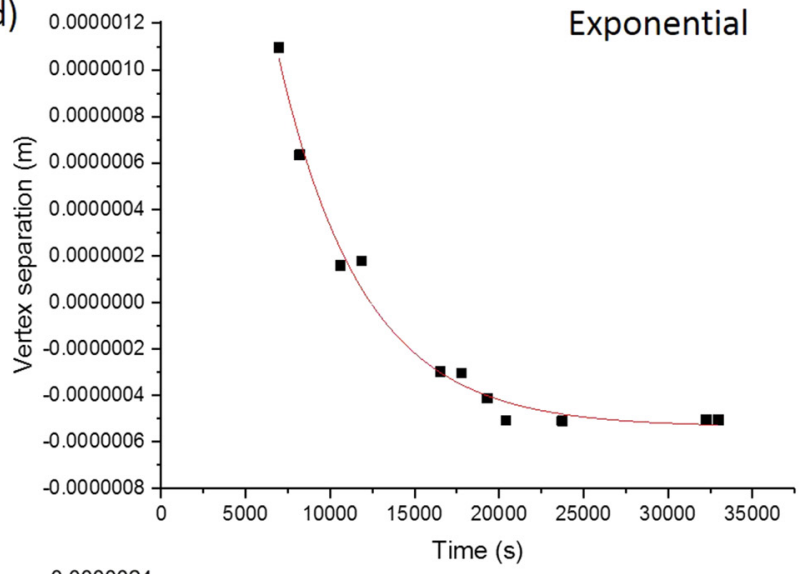

(f)

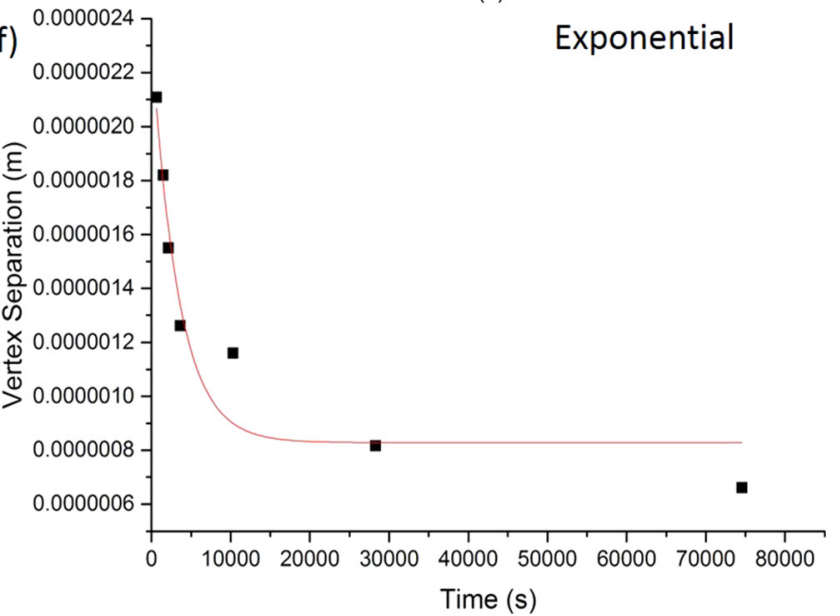

FIG. 8. Relaxation of a 90-degree wall in $\mathrm{BaTiO}_{3}$ at $\mathrm{T}=90^{\circ} \mathrm{C}$; data are fitted to (a) a power law with exponent of 0.25 and (b) an exponential decay with time $\tau=30$ s; data from Ref. 92; (middle) relaxation of domains in Ref. 1, with data fitted to both (c) power-law and (d) exponential decay; (bottom) Time dependence of ferroelectric domain wall relation fitted to (e) power law and (f) exponential. This kind of topological change was reported earlier in nematics, ${ }^{18,119}$ where in plan view it is disconnecting and reconnecting of one-dimensional strings and is thought to occur in cosmological string theory.

in ferroelectric switching ${ }^{31,84-87}$ and hence that the switching dynamics are not dominated by reversal of nano-scale ferroelectric domains within these blocks, i.e., are not described by the Landau-Lifshitz-Kittel domain model. ${ }^{88,89}$ The LandauLifshitz-Kittel model is also a linear response model and hence does not describe domain wall folding, which is a highstress phenomenon, ${ }^{1,90}$ such phenomena are also known in liquid crystals. ${ }^{91}$ Figure 11 illustrates this in a three-step ferroelectric switching in ultra-tetragonal $\mathrm{PbTiO}_{3}{ }^{46}$ The role of these ferroelastic blocks or superdomains is analogous to the role of Abrikosov vortices in thin films of Type II superconductors, where the vortex-antivortex pairs on the meso-scale dominates the dynamics of individual Cooper pairs. Reference 92 supports the conclusion ${ }^{26,31}$ that ferroelastic "bundles of ferroelectric stripes constitute the macroscopic polarization."

\section{CONCLUSIONS}

Period doubling and tripling, wrinkling, and folding in ferroelastic/ferroelectric domains support the early nonlinear 
(a)
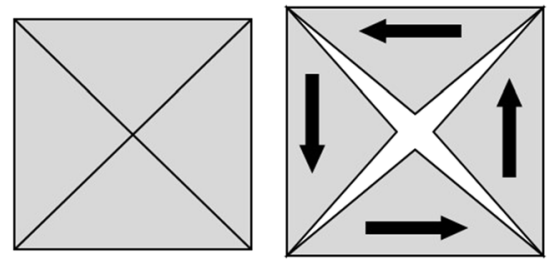

\section{Above $T_{c} \quad$ Below $T_{c}$}

(b)
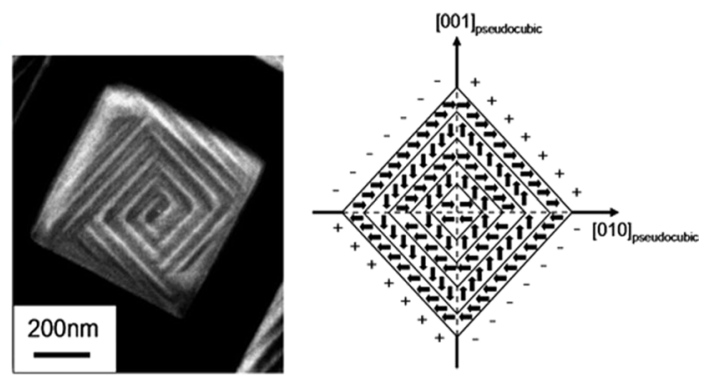

(c)
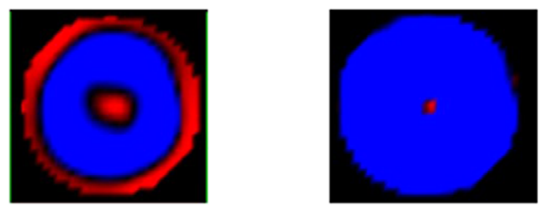

FIG. 9. Top (a)-Diagrams ${ }^{101}$ of closure domains and induced strain (no net polarization); middle (b) - superdomain with net polarization but no net strain. Left side shows experimental data on a spiral domain; right side illustrates a ferroelastic/ferroelectric superdomain; Reprinted with permission from A. Schilling et al., Nano Lett. 9(9), 3359-3364 (2009). Copyright 2009 American Chemical Society. (c) Two bulls-eye domains in 1.0- $\mu \mathrm{m}$ diameter PZT films. ${ }^{80}$ Vortex-antivortex domain pairs (spiral closure domains) can also be triangular; Ref. 103 report the generation of novel equilateral triangular FE closure domains in PZT - three equilateral triangular closure states, consisting of three stripe domain bundles with three 120-degree orientation differences. The observed two closure states had the different rotation directions around the core, clockwise, and counterclockwise.

models of Metaxas et al. ${ }^{70}$ and of Wang and Zhao. ${ }^{71}$ Folding models in elastic thin films seem applicable to thin ferroic domains. Some data exist suggesting the presence of powerlaw relaxation in the jamming of these domains, analogous to ripening, but existing data make it difficult to discriminate between exponential and power-law decay with powers $<1$. This suggests a direction for further work. Other models that still require experimental testing include the predicted $\mathrm{d}^{4 / 9}$ power dependence predicted for folding threshold stress upon film thickness d, ${ }^{93}$ and the conjecture of Aharoni et al. that the wrinkle instability wavelength sets the scale for herringbone patterns. ${ }^{94}$ In addition, the faceting of thin films confined to circular geometries ${ }^{95,96}$ has also been described in terms of wrinkling. ${ }^{97}$ We emphasize for the readers that ferroelastic domains are of great importance not just in ferroelectrics, but in high-Tc superconductors, where so far they have always been found to satisfy the Landau-Lifshitz-Kittel law (square root dependence of domain width versus film thickness), from a few nm to ca. a micron. ${ }^{98}$ Finally, in the context of period doubling and tripling, Roytburd et al. ${ }^{98}$ have recently stressed in a comprehensive review on ferroelastics, which there may be situations in which two different kinds of domains are simultaneously present in quasi- equilibrium, due to boundary conditions, and these do not involve nonlinear doubling or tripling. And Jang's laboratory has shown that the Landau-Lifshitz-Kittel law can fail in nanostructures due to surface layers. ${ }^{99}$ Moreover, Noheda's group has shown that superdomains in $\mathrm{PbSr}_{\mathrm{x}} \mathrm{Ti}_{1-\mathrm{x}} \mathrm{O}_{3}$ can dominate ferroelectric switching. ${ }^{100}$ These in-plane superdomains are strain-neutral, like those in the right-hand side of Fig. 1. It was found that fourfold vertex closure domains in this material are unstable, unlike those in $\mathrm{BaTiO}_{3}{ }^{32,101,102}$ or PZT,${ }^{13}$ in agreement with the clock-model predictions of Srolovitz and Scott. ${ }^{51}$ When treating in the clock model the domain walls, rather than treating the polarization itself, ${ }^{52}$ it is clear that bundle-domain switching, which can be described as correlated motion of defects, ${ }^{86}$ so that the ferroelastic switching indeed dominates the polarization switching, in analogy to the Kosterlitz-Thouless dominancy in thin superconducting films.

Other ferroelectric/ferroelastic systems in which domain motion has been carefully studied include $\mathrm{Bi}_{2} \mathrm{WO}_{6}$, in which the very high ferroelastic mobility is notable. ${ }^{103}$

Regarding power-law versus exponential relaxation, existing data leave this question as moot, suggesting that power laws dominate at long times and exponentials at short times. $^{21,56,104}$

Ferroelectric memories are now rapidly replacing magnetic stripe cards for train and subway access, for cash points ("e-money"), employee identification cards, convenience store sales, luggage lockers, etc., where they are faster, cheaper, and contact-free ${ }^{105}$ (proximity devices which can be read without removal from a wallet). It may be useful to recognize that the active material in use at present in these cards (from, for example, the SONY-family FeliCa or the Suica companies) use ferroelastic-ferroelectrics for which the switching mechanisms discussed above are operative. Other applications of multiferroic domains include the strong coupling of magnetic and ferroelectric walls through their mutual interaction with ferroelastic walls. Van der Wiele et al. ${ }^{59}$ and Allwood et al. ${ }^{106}$ show that the strong coupling of magnetic domain walls onto straight ferroelastic boundaries of a ferroelectric layer enables full and reversible electric-field control of magnetic domain wall motion.

In the context of this paper, the questions for commercial devices are how the ferroelastic properties might limit lateral cell size, switching speed, or reproducibility of switched charge. Perhaps more immediate concern is the role of ferroelastic domains on the performance (either enhancement or degradation of lifetimes) of new solar cell materials. ${ }^{107}$ These may limit performance or provide degradation. Other aspects of ferroelastic domains of current device interest include their role in metal-insulator transitions in materials such as $\mathrm{VO}_{2}{ }^{108}$ It has been known for many years that ferroelastic domains increase toughening in PZT ceramics. ${ }^{109}$ We encourage readers also to take advantage and implement the correlated switching in bundle domains for novel technologies, including those related to domain-wall engineering and in other metaloxide applications.

Readers should keep in mind that many ferroelectrics of recent interest are not ferroelastic (e.g., $\mathrm{YMnO}_{3}$ ). The criterion for ferroelasticity originally suggested by Toledano ${ }^{110}$ 
a
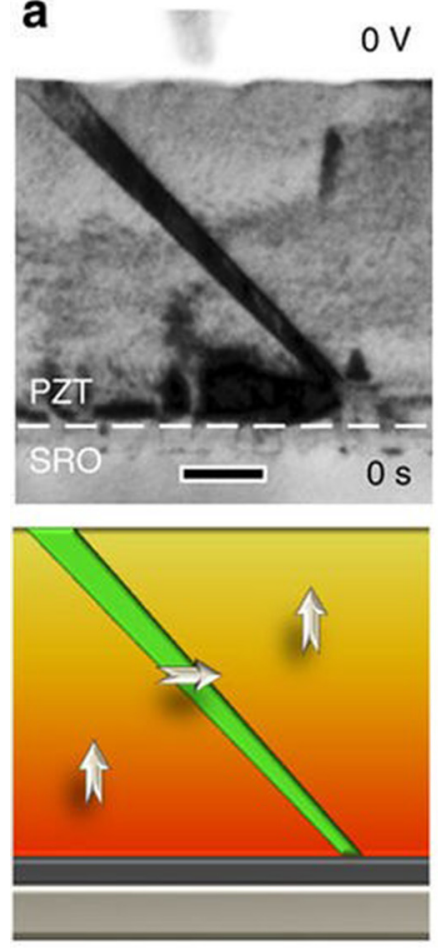

e
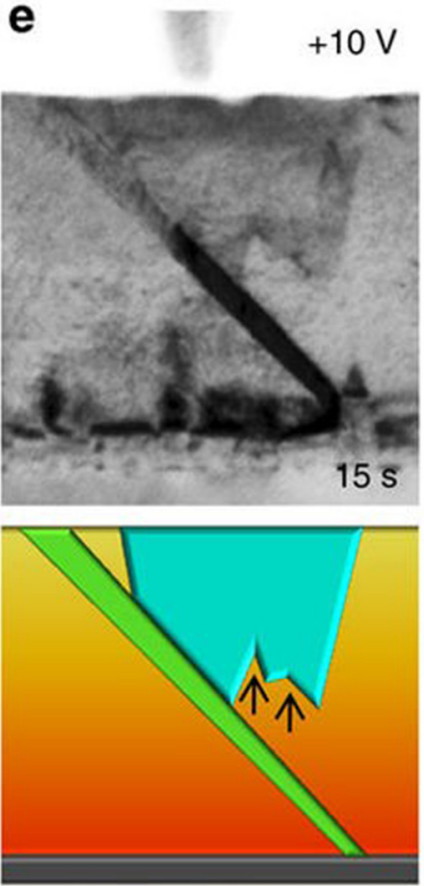

b
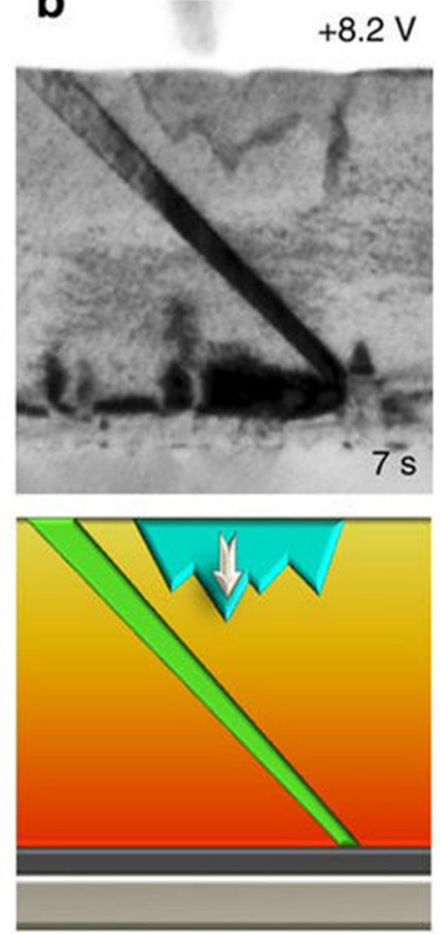

f
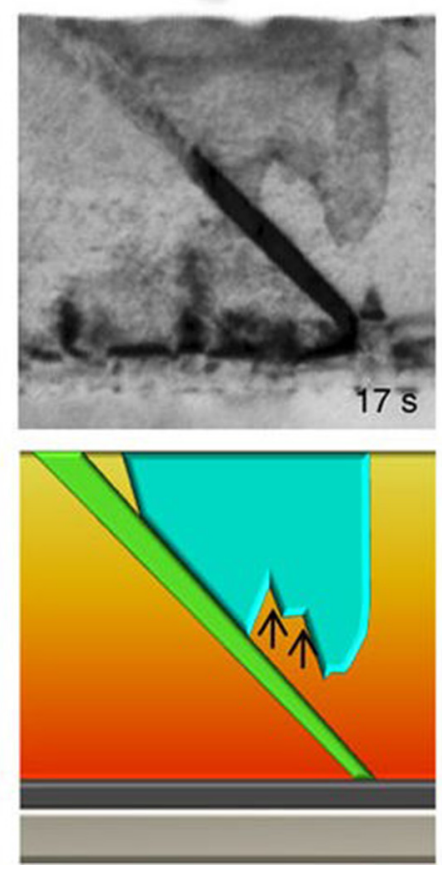

C
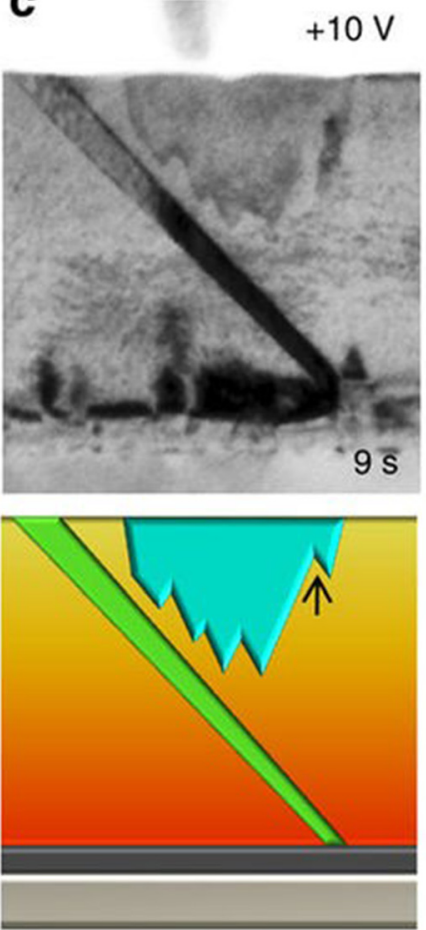

g
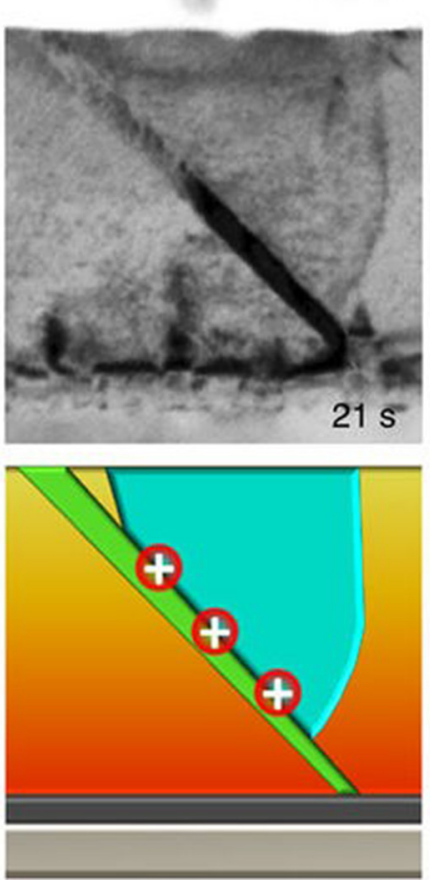
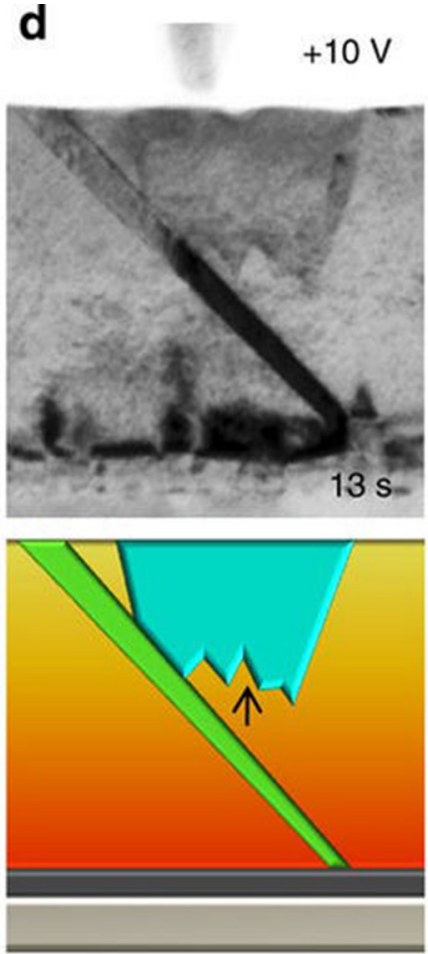

h

$+0 \mathrm{~V}$
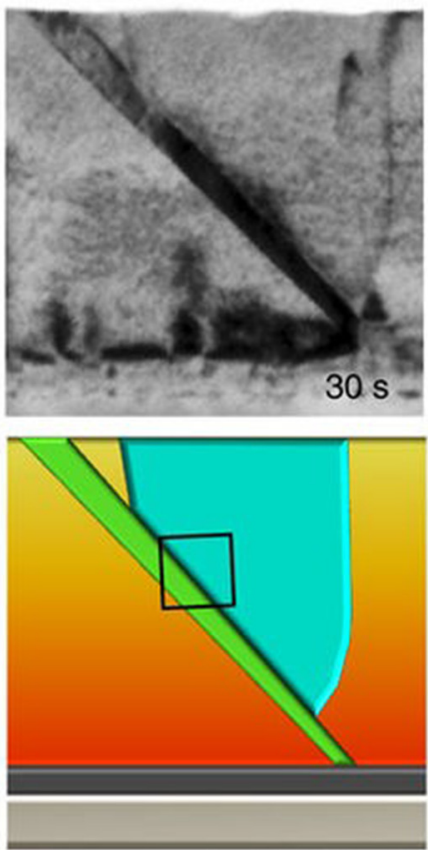

FIG. 10. 180-degree ferroelectric switching that is blocked by a ferroelastic domain wall. ${ }^{45}$ Reprinted with permission from P. Gao et al., Nat. Commun. 4, 2791 (2013). Copyright 2013 Macmillan Publishers Ltd.

was that it is necessary and sufficient that the crystal class change at the ferroelectric transition, such that rhombohedralrhombohedral transitions (e.g., $\mathrm{LiNbO}_{3}$ ) are neither ferroelectric nor orthorhombic-orthorhombic (e.g., $\mathrm{KTiOPO}_{4}$ ). However, this definition requires several exceptions, such as needing hexagonal and trigonal classes to be treated as a single super-class, and not discriminating between Laue Type I and Laue Type II tetragonal structures. ${ }^{111}$ Therefore, we propose a simpler definition that has no exceptions: Ferroelastic transitions are between phases having a different number of nonzero symmetry elements in the fourth-rank elasticity tensor. For example, Laue Type I and II crystals can both be the same tetragonal crystal class but differ in whether $\mathrm{C}_{16}$ is zero. ${ }^{112}$

\section{FUTURE WORK}

Although this review emphasizes net motion of domain walls, we note that oscillation of such walls is also a topic of 


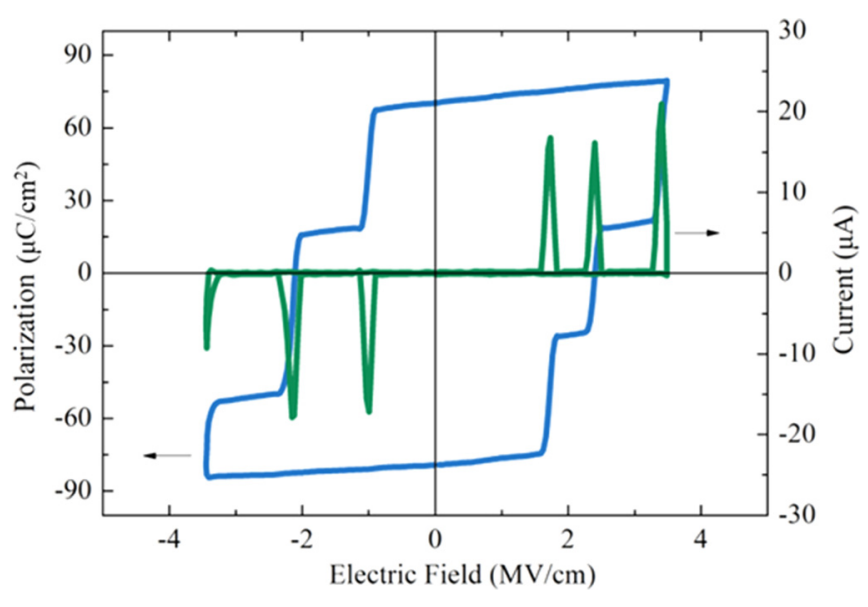

FIG. 11. Polarization switching in ultra-tetragonal $\mathrm{PbTiO}_{3},{ }^{46}$ showing large jumps due to ferroelastic block (superdomain) switching (see also Ref. 45).

current interest. ${ }^{113-115} \mathrm{Chu}$ et al. ${ }^{116}$ have commented that the major contribution to the dielectric response is from the polarization fluctuations on the $90^{\circ}$-domain walls, which are more mobile than those inside the domains. The theory ${ }^{113,114}$ predicts a gap energy in the acoustic phonon/soft-optic mode spectrum at a few $\mathrm{GHz}$, which appears to have been found in ferroelectric tris-sarcosine calcium chloride. ${ }^{115}$ Such lowfrequency overdamped modes can arise from different physical mechanisms, including dynamics of incommensurate domain structures, and in a few cases have been shown ${ }^{117}$ to be diffusive, with linewidth varying as $\mathrm{q}^{2}$, where $\mathrm{q}$ is the momentum transfer; this $\mathrm{q}^{2}$-dependence is a signature of hydrodynamic diffusion and in general supports the basic hydrodynamic model of domain wall motion under stress. ${ }^{1,90}$ Another material of recent interest is $\mathrm{HfO}_{2}$, where ${ }^{120}$ Depner et al. find ferroelastic domains narrower than $1 \mathrm{~nm}$ which are totally absent in bulk. This is in accord with the basic Landau-Lifshitz-Kittel idea that the characteristic length in the problem need not be a macroscopic thickness for all geometries.

In summary, to give a broader perspective on and support for this work, although folding and bent curvature of domain walls in ferroelectric/ferroelastic crystals are a rather new topic, it should not be viewed as controversial or with skepticism: In the context of pure crystallography, both folding and curvature are well known. For example, Ye et al. ${ }^{121}$ comment in their TEM study of bismuth titanate, "domain walls (DWs) lie mainly on the (110) plane, but often fold to the (001) plane. ...The $P_{\mathrm{s}(c)}-180^{\circ}$ DWs observed are irregularly curved." And as early as 1957 Cameron reported "puddle" ferroelectric domains without straight walls in $\mathrm{BaTiO}_{3} .{ }^{122}$

\section{ACKNOWLEDGMENTS}

This work at St. Andrews and at Queens University was supported by EPSRC Grant No. EP/P024637/1; in Lincoln, by the National Science Foundation (NSF) through Materials Research Science and Engineering Center (MRSEC) under Grant No. DMR-1420645; and in Haifa, by Horev Fellowship for Leadership in Science and Technology, supported by the Taub Foundation and the Zuckerman STEM Leadership Program.
${ }^{1}$ J. F. Scott, D. M. Evans, R. S. Katiyar, R. G. P. McQuaid, and J. M. Gregg, "Nonequilibrium ferroelectric-ferroelastic 10-nm nanodomains: Wrinkles, period doubling, and power-law relaxation," J. Phys.: Condens. Matter 29, 304001 (2017).

${ }^{2}$ P. G. Nutting, "A new general law of deformation,” J. Franklin Inst. 191, 679-685 (1921); "A study of elastic viscous deformation," Proc. Am. Soc. Test. Mater. (ASTM) 21, 1162-1171 (1921).

${ }^{3}$ R. Kohlrausch, "Theorie des Elektrischen Ruestanden in der Leiden Flasche," Ann. Phys. Chem. 167, 56-82 (1854).

${ }^{4}$ J. Krug, Kinetic Pattern Formation at Solid Surfaces, Collective Dynamics of Nonlinear and Disordered Systems, edited by G. Radons, W. Just, and P. Haussler (Springer, Berlin, 2005), p. 24.

${ }^{5} \mathrm{~T}$. Michely and J. Krug, Islands, Mounds and Atoms (Springer, Berlin, 2012), p. 184.

${ }^{6}$ P. M. Duxbury and T. J. Pence, Dynamics of Crystal Surfaces and Interfaces (Springer, Berlin, 1997), p. 165.

${ }^{7}$ D. Zhou and M. Kamlah, "Room-temperature creep of soft PZT under static electrical and compressive stress loading," Acta Mater. 54, 1389-1396 (2006).

${ }^{8}$ E. N. C. Andrade, "On the viscous flow in metals and allied phenomena," Proc. R. Soc. A 84, 1-12 (1910).

${ }^{9}$ D. Munz and T. Fett, Ceramics: Mechanical Properties, Failure Behaviour, Materials Selection (Springer-Verlag, Berlin, 1999), p. 275.

${ }^{10} \mathrm{~F}$. S. McClintock and A. S. Argon, Mechanical Behavior of Materials (Addison-Wesley, Reading, MA, 1966), p. 625.

${ }^{11} \mathrm{X}$. Chen, Mechanical Self-Assembly: Science and Applications (Springer, Berlin, 2012), p. 88.

${ }^{12}$ S. W. Chen, H. Guo, K. A. Seu, K. Dumesnil, S. Roy, and S. K. Sinha, "Jamming behavior of domains in a spiral antiferromagnetic system," Phys. Rev. Lett. 110, 217201 (2013).

${ }^{13}$ Y. Ivry, D. P. Chu, J. F. Scott, and C. Durkan, "Flux closure vortexlike domain structures in ferroelectric thin films," Phys. Rev. Lett. 104, 207602 (2010).

${ }^{14}$ S. K. Sinha, "Jamming behavior of domain walls in an antiferromagnetic film," in APS Bulletin, abstract Q7.004 (March 2014).

${ }^{15}$ E. K. H. Salje, "Ferroelastic materials," Ann. Rev. Mater. Res. 42, 1-19 (2012).

${ }^{16}$ E. K. Salje, M. Alexe, S. Kustov, M. C. Weber, J. Schiemer, G. F. Nataf, and J. Kreisel, "Direct observation of polar tweed in $\mathrm{LaAlO}_{3}$," Sci. Rep. 6, 27193 (2016).

${ }^{17}$ A. V. Zvelindovsky, Nanostructured Soft Matter (Springer, Berlin, 2007).

${ }^{18}$ A. V. Zvelindovsky, G. J. A. Sevink, K. S. Lyakhova, and P. Altevogt, "Dynamic density functional theory for sheared polymeric systems," Macromol. Theory Simul. 13, 140-151 (2004).

${ }^{19}$ R. V. Kohn and F. Otto, "Upper bounds on coarsening rates," Commun. Math. Phys. 229, 375-395 (2002).

${ }^{20} \mathrm{C}$. Wagner, "Theorie der Alterung von Niederschlägen durch Umlösen (Ostwald-Reifung) [Theory of the aging of precipitates by dissolutionreprecipitation (Ostwald ripening)]," Z. Elektrochem. 65, 581-591 (1961).

${ }^{21}$ I. M. Lifshitz and V. V. Slyozov, "The kinetics of precipitation from supersaturated solid solutions," J. Phys. Chem. Solids 19, 35-50 (1961).

${ }^{22}$ G. Arlt, H. Dederichs, and R. Herbiet, " $90^{\circ}$-domain wall relaxation in tetragonally distorted ferroelectric ceramics," Ferroelectrics 74, 37-53 (2011).

${ }^{23}$ J. Kubát and M. Rigdahl, "The exponential and power laws of stress relaxation kinetics and a general relation between the activation volume and effective stress," Phys. Status Solidi A 35, 173-180 (1976).

${ }^{24} \mathrm{~W}$. Ostwald, "Studien über die Bildung und Umwandlung fester Körper [Studies on the formation and transformation of solid bodies]," Z. Phys. Chem. 22, 289-330 (1897).

${ }^{25}$ K. Khakalo, K. Baumgarten, B. P. Tigheb, and A. Puisto, "Coarsening and mechanics in the bubble model for wet foams," e-print arXiv:1706.03932.

${ }^{26}$ Y. Ivry, C. Durkan, D. P. Chu, and J. F. Scott, "Nano-domain pinning by extended structural defects,” Adv. Funct. Mater. 24, 5567-5574 (2014).

${ }^{27}$ J. A. Hooton and W. J. Merz, "Etch patterns and ferroelectric domains in $\mathrm{BaTiO}_{3}$ single crystals," Phys. Rev. 98, 409-413 (1955).

${ }^{28}$ D. M. Evans, A. Schilling, A. Kumar, D. Sanchez, N. Ortega, M. Arredondo, R. S. Katiyar, J. M. Gregg, and J. F. Scott, "Magnetic switching of ferroelectric domains at room temperature in a new multiferroic," Nat. Commun. 4, 1534-1540 (2013).

${ }^{29}$ Q. H. Wang, Y. J. Yin, H. M. Xie, J. Liu, W. Yang, P. Chen, and Q. Zhang, "Buckling modes of polymer membranes restricted by metal wires," Soft Matter 7, 2888-2894 (2011). 
${ }^{30}$ B. Li, Y.-P. Can, X.-Q. Feng, and H.-J. Gao, "Mechanics of morphological instabilities and surface wrinkling in soft materials: A review," Soft Matter 8, 5728-5745 (2012).

${ }^{31}$ Y. Ivry, D. P. Chu, and C. Durkan, "Bundles of polytwins as meta-elastic domains in the thin polycrystalline simple multi-ferroic system PZT," Nanotechnology 21, 065702 (2010).

${ }^{32}$ R. G. McQuaid, A. Gruverman, J. F. Scott, and J. M. Gregg, "Exploring vertex interactions in ferroelectric flux-closure domains," Nano Lett. 14, 4230-4237 (2014)

${ }^{33}$ A. Schilling, S. Prosandeev, R. G. P. Mc Quaid, L. Bellaiche, J. F. Scott, and J. M. Gregg, "Shape-induced phase transition of domain patterns in ferroelectric platelets,” Phys. Rev. B 84, 064110 (2011).

${ }^{34}$ R. Ahluwalia, N. Ng, A. Schilling, R. McQuaid, D. J. Srolovitz, D. Evans, J. M. Gregg, and J. F. Scott, "Manipulating ferroelectric domains in nano-structures under electron beams," Phys. Rev. Lett. 111, 165702 (2013)

${ }^{35}$ N. Ng, R. Ahluwalia, A. Kumar, D. J. Srolovitz, P. Chandra, and J. F. Scott, "Electron-beam driven relaxation oscillations in ferroelectric nanodisks," Appl. Phys. Lett. 107, 152902 (2015).

${ }^{36}$ T. Tybell, P. Paruch, T. Giamarchi, and J. M. Triscone, "Domain wall creep in epitaxial ferroelectric $\mathrm{Pb}\left(\mathrm{Zr}_{0.2} \mathrm{Ti}_{0.8}\right) \mathrm{O}_{3}$ thin films," Phys. Rev. Lett. 89, 097601 (2002)

${ }^{37}$ J. Y. Jo, S. M. Yang, T. H. Kim, and H. N. Lee, "Nonlinear dynamics of domain-wall propagation in epitaxial ferroelectric thin films," Phys. Rev. Lett. 102, 045701 (2009).

${ }^{38}$ L. A. Bursill and P. J. Lin, "Electron microscopic studies of ferroelectric crystals," Ferroelectrics 70, 191-203 (1986).

${ }^{39}$ D. Viehland, Z. Xu, and W.-H. Huang, "Structure-property relationships in strontium barium niobate I. Needle-like nanopolar domains and the metastably-locked incommensurate structure," Philos. Mag. A 71 , 205-217 (1995)

${ }^{40}$ A. Butykai, S. Bordacs, I. Kezsmarki, V. Tsurkan, A. Loidl, J. Doring, P. Milde, S. C. Kehr, and L. M. Eng, "Characteristics of ferroelectricferroelastic domains in Neel-type skyrmion host $\mathrm{GaV}_{4} \mathrm{~S}_{8}$, " 15, 44663 (2017)

${ }^{41}$ H. Muller, W. Kockelmann, and D. Johrendt, "The magnetic structure and electronic ground states of Mott insulators $\mathrm{GeV}_{4} \mathrm{~S}_{8}$ and $\mathrm{GaV}_{4} \mathrm{~S}_{8}$," Chem. Mater. 18, 2174-2180 (2006)

${ }^{42}$ S. H. Yun, C.-S. Woo, G.-Y. Kim, P. Sharma, J. H. Lee1, K. H. Chu, J. H. Song, S.-Y. Chung, J. Seidel, S.-Y. Choi, and C.-H. Yang, "Ferroelastic twin structures in epitaxial $\mathrm{WO}_{3}$ thin films," Appl. Phys. Lett. 107, 252904 (2015).

${ }^{43}$ V. Janovec, “A symmetry approach to domain structures," Ferroelectrics 12, 43-53 (1976).

${ }^{44}$ H.-T. Jeong, S.-Y. Jeong, W. T. Kim, and D. H. Kim, "The experimental evidence on the existence of fourfold ferroelastic domain wall," J. Phys. Soc. Jpn. 69, 306-308 (2000).

${ }^{45}$ P. Gao, J. Britson, J. R. Jokisaari, C. T. Nelson, S.-H. Baek, W. Yiran, C. B. Eom, L. Q. Chen, and X. Q. Pan, "Atomic-scale mechanisms of ferroelastic domain-wall-mediated ferroelectric switching," Nat. Commun. 4, 2791 (2013)

${ }^{46}$ L. X. Zhang, J. Chen, O. Diéguez, J. L. Cao, L. L. Fan, Z. Pan, Y. L. Wang, J. G. Wang, M. Kim, S. Q. Deng, J. A. Wang, H. H. Wang, J. X. Deng, J. F. Scott, and X. R. Xin, "Giant polarization in super-tetragonal ferroelectric thin through interphase strain," Science (in press).

${ }^{47}$ J. M. Kosterlitz and D. J. Thouless, "Ordering, metastability and phase transitions in two-dimensional systems," J. Phys. C: Solid State Phys. 6, 1181-1202 (1973).

${ }^{48}$ T. A. Engstrom and J. M. Schwarz, "Surface creasing of soft elastic continua as a Kosterlitz-Thouless transition," EPL 118, 56005 (2017).

${ }^{49}$ O. Borisenko, V. Chelnokov, G. Cortese, G. R. Fiore, M. Gravina, and A. Papa, "Phase transitions in two-dimensional $\mathrm{Z}(\mathrm{N})$ vector models for $\mathrm{N}>$ 4,” Phys. Rev. E 85, 021114 (2012).

${ }^{50}$ J. F. Scott, "Extended defects in nano-ferroelectrics," in Topological Structures in Ferroelectric Materials: Domain Walls, Vortices, and Skyrmions, Springer Series in Materials Science 228 (Springer, Heidelberg, 2016), especially p. 143 and Ref. 21.

${ }^{51}$ D. J. Srolovitz and J. F. Scott, "Clock-model description of incommensurate ferroelectric films and of nematic liquid-crystal films," Phys. Rev. B 34, 1815-1819 (1986).

${ }^{52}$ L. A. Delimova, E. V. Guschina, D. S. Seregin, K. A. Vorotilov, and A. S. Sigov, "Unexpected behavior of transient current in thin PZT films caused by grain-boundary conduction," J. Appl. Phys. 121, 224104 (2017)
${ }^{53}$ E. K. H. Salje, "Multiferroic domain boundaries as active memory devices: Trajectories towards domain boundary engineering," Chem. Phys. Chem. 11, 940-950 (2010).

${ }^{54}$ E. K. H. Salje and M. A. Carpenter, "Domain glasses: Twin planes, Bloch lines, and Bloch points," Phys. Status Solidi B 252, 2639-2648 (2015).

${ }^{55}$ E. K. H. Salje, X. Wang, X. Ding, and J. F. Scott, "Ultrafast switching in ferroelectrics by supersonic kink movements," Adv. Funct. Mater. 27, 00367-00372 (2017).

${ }^{56}$ S. Puchberger, V. Soprunyuk, W. Schranz, A. Tröster, K. Roleder, A. Majchrowski, M. A. Carpenter, and E. K. H. Salje, "The noise of many needles: Jerky domain wall propagation in $\mathrm{PbZrO}_{3}$ and $\mathrm{LaAlO}_{3}$," APL Mater. 5, 046102 (2017).

${ }^{57}$ R. Metzler and J. Klafter, "From stretched exponential to inverse powerlaw: Fractional dynamics, Cole-Cole relaxation processes, and beyond," J. Non-Cryst. Solids 305, 81-87 (2002)

${ }^{58}$ E. C. de Oliveira, F. Mainardi, and J. Vaz, "Models based on MittagLeffler functions for anomalous relaxation in dielectrics," Eur. Phys. J. (Spec. Top.) 193, 161-171 (2011).

${ }^{59}$ B. Van de Wiele, J. Leliaert, K. J. A. Franke, and J. van Djiken, "Electricfield-driven dynamics of magnetic domain walls in magnetic nanowires patterned on ferroelectric domains," New J. Phys. 18, 033027 (2016).

${ }^{60} \mathrm{~K}$. Weron, "A probabilistic mechanism hidden behind the universal power law for dielectric relaxation," Report No. IC91/246, International Atomic Energy Agency and UNESCO, International Centre for Theoretical Physics, Trieste, Italy, 1991.

${ }^{61} \mathrm{~K}$. Weron and A. Jurlewicz, "Two forms of self-similarity as a fundamental feature of the power-law dielectric response," J. Phys. A: Math. Gen. 26, 395-410 (1993).

${ }^{62}$ A. A. Stanislavsky and K. Weron, "Stochastic tools hidden behind the empirical dielectric relaxation laws," Rep. Prog. Phys. 80, 036001 (2017).

${ }^{63}$ B. G. Buelfinger, De Solidorum Resistentia Specimen (Commentary Academy of Sciences, Petroli, 1729), pp. 164-181.

${ }^{64}$ L. C. Sampaio, M. P. de Albuquerque, and F. S. de Menezes, "Magnetic relaxation and formation of magnetic domains in ultrathin films with perpendicular anisotropy," Phys. Rev. B 54, 6465-6467 (1996).

${ }^{65}$ Y. J. Gu, M. L. Li, A. N. Morozovska, Y. Wang, E. A. Eliseev, V. Gopalan, and L. Q. Chen, "Non-Ising character of a ferroelectric wall arises from a flexoelectric effect," e-print arXiv:1401.0194.

${ }^{66}$ M. L. Li, Y. J. Gu, L. Q. Chen, and W. H. Duan, "First-principles study of 180 domain walls in $\mathrm{BaTiO}_{3}$," Phys. Rev. B 90, 054106 (2014).

${ }^{67}$ J. Guyonnet, Ferroelectric Domain Walls: Statics, Dynamics, and Functionalities Revealed by Atomic Force Microscopy (Springer, Heidelberg, 2014).

${ }^{68}$ G. Catalan, J. F. Scott, A. Schilling, and J. M. Gregg, "Wall thickness dependence of the scaling law for ferroelectric stripe domains," J. Phys.: Condens. Matter 19, 022201 (2007).

${ }^{69}$ G. Catalan, J. Seidel, R. Ramesh, and J. F. Scott, "Domain wall nanoelectronics," Rev. Mod. Phys. 84, 119-156 (2012).

${ }^{70}$ P. J. Metaxas, J. P. Jamet, A. Mougin, M. Cormier, J. Ferré, V. Baltz, B. Rodmacq, B. Dieny, and R. L. Stamps, "Creep and flow regimes of magnetic domain-wall motion in ultrathin $\mathrm{Pt} / \mathrm{Co} / \mathrm{Pt}$ films with perpendicular anisotropy," Phys. Rev. Lett. 99, 217208 (2007).

${ }^{71} \mathrm{Q}$. Wang and X. H. Zhao, "A three-dimensional phase diagram of growth-induced surface instabilities," Sci. Rep. 5, 8887 (2015).

${ }^{72}$ G. Catalan, A. Schilling, J. F. Scott, and J. M. Gregg, "Domains in threedimensional ferroelectric nanostructures: Theory and experiment," J. Phys.: Condens. Matter 19, 132201 (2007).

${ }^{73}$ F. Brau, H. Vandeparre, A. Sabbah, C. Poulard, A. Boudaoud, and P. Damman, "Multiple-length-scale elastic instability mimics parametric resonance of nonlinear oscillators," Nat. Phys. 7, 56-60 (2011).

${ }^{74}$ F. Brau, P. Damman, H. Diamant, and T. A. Witten, "Wrinkle-to-fold transitions," Soft Matter 9, 8177-8186 (2013).

${ }^{75}$ Y. Ivry, N. Wang, D. P. Chu, and C. Durkan, " $90^{\circ}$ domain dynamics and relaxation in thin ferroelectric/ferroelastic films," Phys. Rev. B 81, 174118 (2010).

${ }^{76}$ C. S. Ganpule, A. L. Roytburd, V. Nagarajan, B. K. Hill, S. B. Ogale, E. D. Williams, and R. Ramesh, "Polar relaxation kinetics and 180-degree domain wall dynamics in ferroelectric thin films," Phys. Rev. B 65, 014101 (2001).

${ }^{77}$ L. H. Hamedi, M. Guilloux-Viry, and G. Perrin, "Heteroepitaxy and growth of PZT thin films," Thin Solid Films 352, 66-72 (1999).

${ }^{78}$ H. Béa, B. Ziegler, M. Bibes, A. Barthélémy, and P. Paruch, "Nanoscale polarization switching mechanisms in multiferroic $\mathrm{BiFeO}_{3}$ thin films," J. Phys.: Condens. Matter 23, 142201 (2011). 
${ }^{79}$ P. C. Hohenberg and A. P. Krekhov, "An introduction to GinzburgLandau theory of phase transitions and nonequilibrium patterns," Phys. Rep. 572, 1-42 (2015).

${ }^{80}$ A. Gruverman, D. Wu, H. J. Fan, I. Vrejou, M. Alexe, R. J. Harrison, and J. F. Scott, "Vortex ferroelectric domains," J. Phys.: Condens. Matter 20, 342201 (2008).

${ }^{81}$ M. Dawber, D. J. Jung, and J. F. Scott, "Perimeter effect in very small ferroelectrics," Appl. Phys. Lett. 82, 436-438 (2003).

${ }^{82}$ S. R. Bakaul, B. F. Miao, W. Lin, W. Hu, A. David, H. F. Ding, and T. $\mathrm{Wu}$, "Domain-related origin of magnetic relaxation in compressively strained manganite thin films," Appl. Phys. Lett. 101, 012408 (2012).

${ }^{83}$ H. W. Jang, D. Ortiz, S.-H. Baek, C. M. Folkman, R. R. Das, P. Shafer, Y.-B. Chen, C. T. Nelson, X.-Q. Pan, R. Ramesh, and C.-B. Eom, "Domain Engineering for Enhanced Ferroelectric Properties of Epitaxial (001) BiFeO Thin Films," Adv. Mater. 21, 817-821 (2009).

${ }^{84}$ L. J. McGilly and J. M. Gregg, "Scaling of superdomain bands in ferroelectric dots," Appl. Phys. Lett. 98, 132902 (2011)

${ }^{85}$ L. J. McGilly, T. L. Burnett, A. Schilling, M. G. Cain, and J. M. Gregg, "Domain annihilation due to temperature and thickness gradients in single crystal $\mathrm{BaTiO}_{3}$," Phys. Rev. B 85, 054113 (2012).

${ }^{86}$ C. Durkan, A. Hershkovitz, D. Chu, J. F. Scott, and Y. Ivry, "Towards resolving Landauer's paradox through direct observation of multiscale ferroelastic-ferroelectric interplay," e-print arXiv:1608.03890v1 [condmat.mtrl-sci].

${ }^{87}$ Y. Ivry, J. F. Scott, E. K. H. Salje, and C. Durkan, "Nucleation, growth, and control of ferroelectric-ferroelastic domains in thin polycrystalline films," Phys. Rev. B 86, 205428 (2012).

${ }^{88}$ L. D. Landau and E. M. Lifshitz, "On the theory of dispersion of magnetic permeability in ferromagnetic bodies," Phys. Z. Sowjetunion 8, 101-114 (1935).

${ }^{89}$ C. Kittel, "Physical theory of ferromagnetic domains," Rev. Mod. Phys. 21, 541-583 (1949).

${ }^{90}$ J. F. Scott, D. M. Evans, J. M. Gregg, and A. Gruverman, "Hydrodynamics of domain walls in ferroelectrics and multiferroics: Impact on memory devices," Appl. Phys. Lett. 109, 042901 (2016).

${ }^{91}$ L. Blinov, "Domain instabilities in liquid crystals," J. Phys. Colloq. 40(C3), 247-258 (1979).

${ }^{92}$ D. Y. He, X. R. Xing, L. J. Qiao, and A. A. Volinsky, "Temperature change effect on $\mathrm{BaTiO}_{3}$ single-crystal surface potential around domain walls," Appl. Surf. Sci. 311, 837-841 (2014).

${ }^{93}$ D. P. Holmes and A. J. Crosby, "Draping films: A wrinkle to fold transition," Phys. Rev. Lett. 105, 038303 (2010).

${ }^{94}$ H. Aharoni, D. V. Todorova, O. Albarrán, L. Goehring, R. D. Kamien, and E. Katifori, “The smectic order of wrinkles," Nat. Commun. 8, 15809 (2017).

${ }^{95}$ I. Lukyanchuk, P. Sharma, T. Nakajima, S. Okamura, J. F. Scott, and A. Gruverman, "High-symmetry polarization domains in low-symmetry ferroelectrics," Nano Lett. 14, 6931-6935 (2014).

${ }^{96}$ L. Baudry, I. Luk'yanchuk, and J. F. Scott, "Polarization vortex domains induced by switching electric field in ferroelectric films with circular electrodes," Phys. Rev. B 90, 024102 (2014).

${ }^{97}$ J. H. Na, S. U. Kim, Y. J. Sohn, and S. D. Lee, "Self-organized wrinkling patterns of a liquid crystalline polymer in surface wetting confinement," Soft Matter 11, 4788-4792 (2015)

${ }^{98}$ A. L. Roytburd, J. Ouyang, and A. Artemov, "Polydomain structures in ferroelectric and ferroelastic epitaxial films," J. Phys.: Condens. Matter 29, 163001 (2017)

${ }^{99}$ J. Y. Son, S. W. Song, J.-H. Lee, and H. M. Jang, “Anomalous domain periodicity observed in ferroelectric $\mathrm{PbTiO}_{3}$ nanodots having $180^{\circ}$ stripe domains," Sci. Rep. 6, 26644 (2016).

${ }^{100}$ S. Matzen, O. Nesterov, G. Rispens, J. A. Heuver, M. Biegalski, H. M. Christen, and B. Noheda, "Super switching and control of in-plane ferroelectric nanodomains in strained thin films," Nat. Commun. 5, 4415-4418 (2014).

${ }^{101}$ A. Schilling, D. Byrne, G. Catalan, K. G. Webber, Y. A. Genenko, G. S. Wu, J. F. Scott, and J. M. Gregg, "Domains in ferroelectric nanodots," Nano Lett. 9(9), 3359-3364 (2009).
${ }^{102}$ S. M. Yang, Y. J. Shin, T. W. Noh, Y. Ehara, H. Funakubo, J.-G. Yoon, and J. F. Scott, "Equilateral triangular ferroelectric closure domains in (111)-oriented epitaxial $\mathrm{Pb}(\mathrm{Zr}, \mathrm{Ti}) \mathrm{O}_{3}$ thin films," APS Bull. 58, C21.007 (2013).

${ }^{103}$ C. S. Wang, X. X. Ke, J. J. Wang, R. R. Liang, Z. L. Luo, T. A. Yu, Y. Di, Q. T. Zhang, J. Wang, X. F. Han, G. Van Tendeloo, L. Q. Chen, C. W. Nan, R. Ramesh, and J. X. Zhang, "Ferroelastic switching in a layered-perovskite thin film," Nat. Commun. 7, 10636 (2016).

${ }^{104}$ Y.-C. Chen, C.-H. Ko, Y.-C. Huang, J.-C. Yang, and Y. Chu, "Domain relaxation dynamics in epitaxial $\mathrm{BiFeO}_{3}$ films: Role of surface charges," J. Appl. Phys. 112, 052017 (2012).

${ }^{105}$ J. F. Scott, "A short history of ferroelectric random access memories (FRAMs)," in Proceedings of International Meeting on Nonvolatile Memories, edited by S. Sze (Taiwan University Press, Taipei, 2017), pp. 75-81.

${ }^{106}$ D. A. Allwood, G. Xiong, C. C. Faulkner, D. Atkinson, D. Petit, and R. P. Cowburn, "Magnetic Domain-Wall Logic," Science 309, 1688-1692 (2005).

${ }^{107}$ E. Strelcov, Q. F. Dong, T. Lee, J. S. Chae, Y. C. Shao, Y. H. Deng, A. Gruverman, J. S. Huang, and A. Centrone, " $\mathrm{CH}_{3} \mathrm{NH}_{3} \mathrm{PbI}_{3}$ perovskites: Ferroelasticity revealed," Sci. Adv. 3, e1602165 (2017).

${ }^{108}$ A. Tselev, E. Strelcov, I. A. Luk'yanchuk, J. D. Budai, J. Z. Tischler, I. N. Ivanov, K. Jones, R. Proksch, S. V. Kalinin, and A. Kolmakov, "Interplay between ferroelastic and metal-insulator phase transitions in strained quasi-two-dimensional $\mathrm{VO}_{2}$ nanoplatelets," Nano Lett. 10, 2003-2011 (2010)

${ }^{109} \mathrm{~K}$. Mehta and A. V. Virka, "Fracture mechanisms in ferroelectricferroelastic lead zirconate titanate $(\mathrm{Zr}: \mathrm{Ti}=0.54: 0.46)$ ceramics," J. Am. Ceram. Soc. 73, 567-574 (1990).

${ }^{110}$ J.-C. Toledano, "La Ferroelastice," Ann. Telecommun. 29, 249-270 (1974).

${ }^{111}$ J. F. Scott, "Flexoelectric spectroscopy," J. Phys.: Condens. Matter 25, 331001 (2013)

${ }^{112}$ Y. Liu, J. F. Scott, and B. Dkhil, "Some strategies for improving caloric responses with ferroelectrics," APL Mater. 4, 064109 (2016).

${ }^{113}$ R. T. Brierley and P. B. Littlewood, "Domain wall fluctuations in ferroelectrics coupled to strain,” Phys. Rev. B 89, 184104 (2014).

${ }^{114}$ A. Pakhmanov, I. Luk'yanchuk, and A. Sidorkin, "Frequency dependence of the dielectric permittivity in ferroelectric thin films with $180^{\circ}$ domain structure," Ferroelectrics 444, 177-182 (2013).

${ }^{115}$ R. Mackeviciute, M. Ivanov, J. Banys, N. Novak, Z. Kutnjak, M. Wencka, and J. F. Scott, "The perfect soft-mode: Giant phonon instability in a ferroelectric," J. Phys.: Condens. Matter 25, 212201 (2013).

${ }^{116}$ P. Chu, D. P. Chen, Y. L. Wang, Y. L. Xie, Z. B. Yan, J. G. Wan, J. M. Liu, and J. Y. Li, "Kinetics of $90^{\circ}$ domain wall motions and high frequency mesoscopic dielectric response in strained ferroelectrics: A phasefield simulation," Sci. Rep. 4, 5007 (2014).

${ }^{117}$ W. F. Oliver, J. F. Scott, S. A. Lee, and S. A. Lindsay, "A quasi-elastic light-scattering study of the $1 \mathrm{q}$ to $2 \mathrm{q}$ transition within the incommensurate phase of $\mathrm{Ba}_{2} \mathrm{NaNb}_{5} \mathrm{O}_{15}$," J. Phys.: Condens. Matter 2, 2465-2471 (1990).

${ }^{118}$ I. Chuang, R. Durrer, N. Turok, and B. Yurke, "Cosmology in the laboratory: Defect dynamics in liquid crystals," Science 251, 1336-1342 (1991).

${ }^{119}$ I. I. Chuang, B. Yurke, A. N. Pargellis, and N. Turok, "Coarsening dynamics in uniaxial nematic liquid crystals," Phys. Rev. E 47, 3343-3356 (1993).

${ }^{120}$ S. W. Depner, N. D. Cultrara, K. E. Farley, Y. L. Qin, and S. Banerjee, "Ferroelastic domain organization and precursor control of size in solutiongrown hafnium dioxide nanorods," ACS Nano 8, 4678-4688 (2014).

${ }^{121}$ W. Ye, C. Lu, Y. Zhang, and Y. Zhou, "Types and configurations of domain walls in ferroelectric $\mathrm{Bi}_{4} \mathrm{Ti}_{3} \mathrm{O}_{12}$ single crystals," J. Appl. Crystallogr. 48, 1080-1088 (2015).

${ }^{122}$ D. P. Cameron, "Domain orientation in barium titanate single crystals," IBM J. Res. Dev. 1, 2-7 (1957).

${ }^{123}$ Y. Ivry, D. Chu, J. F. Scott, and C. Durkan, "Domains beyond grain boundaries," Adv. Funct. Mater. 21, 1746 (2011) 